\title{
Estimating the Quadratic Covariation Matrix from Noisy Observations: Local Method of Moments and Efficiency
}

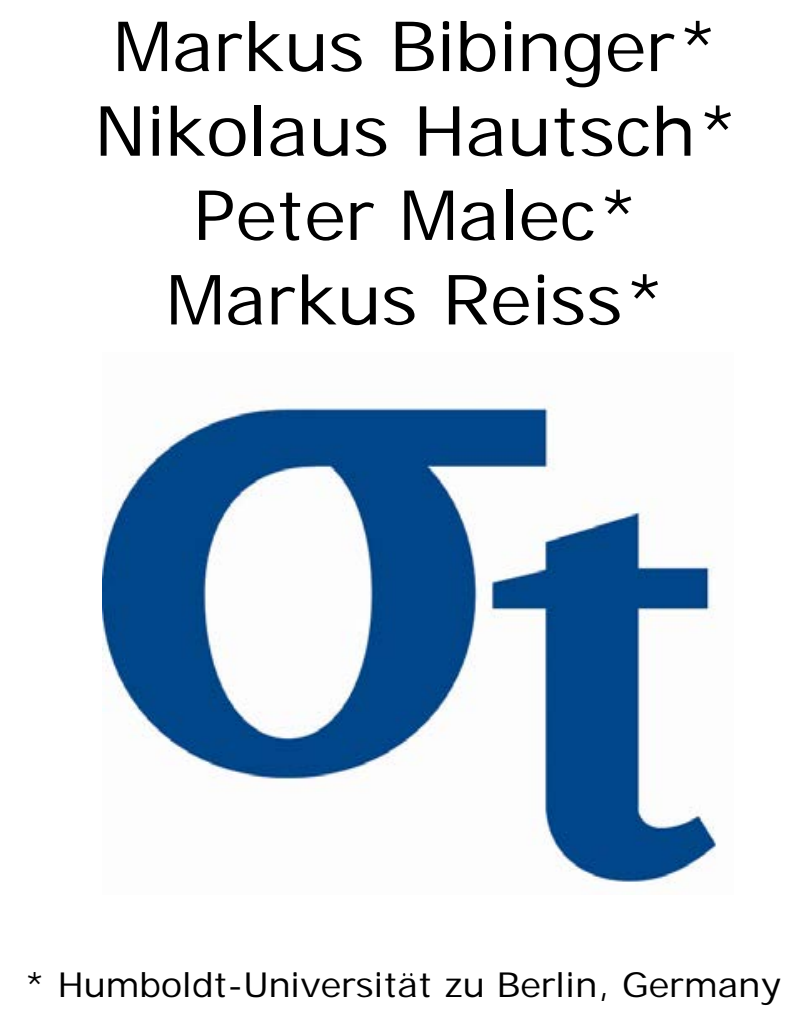

This research was supported by the Deutsche Forschungsgemeinschaft through the SFB 649 "Economic Risk". 


\title{
ESTIMATING THE QUADRATIC COVARIATION MATRIX FROM NOISY OBSERVATIONS: LOCAL METHOD OF MOMENTS AND EFFICIENCY
}

\author{
By Markus Bibinger*, Nikolaus Hautsch*, Peter Malec* \\ AND MaRKus ReISs* \\ Humboldt-Universität zu Berlin
}

\begin{abstract}
An efficient estimator is constructed for the quadratic covariation or integrated covolatility matrix of a multivariate continuous martingale based on noisy and non-synchronous observations under high-frequency asymptotics. Our approach relies on an asymptotically equivalent continuous-time observation model where a local generalised method of moments in the spectral domain turns out to be optimal. Asymptotic semiparametric efficiency is established in the Cramér-Rao sense. Main findings are that non-synchronicity of observation times has no impact on the asymptotics and that major efficiency gains are possible under correlation. Simulations illustrate the finite-sample behaviour.
\end{abstract}

1. Introduction. The estimation of the quadratic variation or integrated volatility of a semi-martingale is a key question, both from a theoretical viewpoint as well as for applications, particularly in finance. Here we treat the multi-dimensional case, where the quadratic covariation or integrated covolatility matrix is the quantity of interest. It turns out that the richer geometry, e.g. due to non-commuting matrices, generates new effects and calls for a deeper mathematical understanding. Covariation estimates are particularly important in various financial applications, for instance, as inputs in portfolio allocation problems, quantification of risk, hedging or asset pricing. The availability of high-frequency data opens up new ways for inference. As the data is typically polluted by observational noise, e.g. by microstructure frictions, estimation in these models is far from obvious and furnishes unexpected results.

\footnotetext{
${ }^{*}$ Financial support from the Deutsche Forschungsgemeinschaft via SFB 649 Ökonomisches Risiko and FOR 1735 Structural Inference in Statistics: Adaptation and Efficiency is gratefully acknowledged.

AMS 2000 subject classifications: Primary 62M10; secondary 62G05; JEL classes: C14, C32, C58, G10.

Keywords and phrases: adaptive estimation, asymptotic equivalence, asynchronous observations, integrated covolatility matrix, quadratic covariation, semiparametric efficiency, microstructure noise, spectral estimation
} 
We focus on the fundamental statistical model where the $d$-dimensional discrete-time process

$$
Y_{i}^{(l)}=X_{t_{i}^{(l)}}^{(l)}+\varepsilon_{i}^{(l)}, 0 \leq i \leq n_{l}, 1 \leq l \leq d,
$$

is observed with the $d$-dimensional continuous martingale

$$
X_{t}=X_{0}+\int_{0}^{t} \Sigma^{1 / 2}(s) d B_{s}, t \in[0,1],
$$

in terms of a $d$-dimensional standard Brownian motion $B$ and the squared (instantaneous or spot) covolatility matrix

$$
\Sigma(t)=\left(\Sigma_{l r}(t)\right)_{1 \leq l, r \leq d} \in \mathbb{R}^{d \times d} .
$$

The signal part $X$ is assumed to be independent of the observation noise $\varepsilon$. The observation errors $\left(\varepsilon_{i}^{(l)}\right), 1 \leq l \leq d, 1 \leq i \leq n_{l}$, are assumed to be mutually independent and centered normal with variances $\eta_{l}^{2}$. The observation times are given via quantile transformations as $t_{i}^{l}=F_{l}^{-1}\left(i / n_{l}\right)$ for some distribution functions $F_{l}$. While this model is certainly an idealisation of many real data situations, its precise analysis delivers a profound understanding and thus serves as a basis for developing procedures in more complex models.

Covariation estimation is a core research topic in current financial econometrics and various approaches exist. Let us mention the quasi-maximumlikelihood method by Aït-Sahalia et al. [1], realised kernels by BarndorffNielsen et al. [3], pre-averaging by Christensen et al. [5] and the local spectral estimator by Bibinger and Reiß [4]. In contrast to the univariate case, however, the asymptotic properties are very involved, difficult to compare and a lower efficiency bound was lacking as a benchmark.

Building on the idea of locally constant approximations, we propose a local method of moments (LMM) estimator in the spectral domain which is shown to be asymptotically efficient. We perform an asymptotic analysis where the sample sizes $n_{1}, \ldots, n_{d}$ tend to infinity. In Section 2 (strong) asymptotic equivalence in Le Cam's sense is established by Theorem 2.4 with the signal-in-white-noise model

$$
d Y_{t}=X_{t} d t+\operatorname{diag}\left(H_{n, l}(t)\right)_{1 \leq l \leq d} d W_{t}, t \in[0,1],
$$

where $W$ is a standard $d$-dimensional Brownian motion independent of $B$ and the local noise level is given by

$$
H_{n, l}(t):=\eta_{l}\left(n_{l} F_{l}^{\prime}(t)\right)^{-1 / 2} .
$$


The imposed regularity condition is that $\Sigma(t)$ is the sum of an $L^{2}$-Sobolev function of regularity $\beta$ and an $L^{2}$-martingale and the size of $\beta$ accommodates for asymptotically separating sample sizes $\left(n_{l}\right)_{1 \leq l \leq d}$.

Let us recall that if two sequences of statistical experiments are asymptotically equivalent, then any statistical procedure in one experiment has a counterpart in the other experiment with the same asymptotic properties for bounded loss functions, see Le Cam and Yang [17] for a thorough introduction. Our proof is constructive such that the procedure that we shall develop for $\left(\mathcal{E}_{1}\right)$ has a concrete counterpart in $\left(\mathcal{E}_{0}\right)$ with the same asymptotic properties.

A remarkable theoretical consequence of this result is that under noise the asynchronicity of the data does not affect the asymptotically efficient procedures (it is of smaller asymptotic order). In model $\left(\mathcal{E}_{1}\right)$ the distribution functions $F_{l}$ only generate a varying local noise level $H_{n, l}(t)$, but the shift between observation times of different processes does not matter. This is in sharp contrast to the noiseless setting where the variance of the HayashiYoshida estimator [12] suffers from errors due to asynchronicity, which carries over to the preaveraged version by Christensen et al. [5] designed for the noisy case.

In Section 3 we consider the continuous-time model $\left(\mathcal{E}_{1}\right)$ and go over to a block-wise constant approximation. Empirical Fourier coefficients yield local spectral statistics $\left(S_{j k}\right)$ in the spirit of Reiß [19]. On each block we apply locally a generalised method of moments, using a weighted sum of the empirical covariance matrices $S_{j k} S_{j k}^{\top} \in \mathbb{R}^{d \times d}$ and a bias correction. The optimal weighting for estimating an entry of the covariation matrix combines in general all entries of $S_{j k} S_{j k}^{\top}$. The non-commutativity of different Fisher information matrices then implies in particular that the volatility estimation for one coordinate process $X^{(l)}$ gains in efficiency when using data for all other potentially correlated processes $X^{(r)}$, see Sections 4.2 and 5 for details and the improvement with respect to the approach in Bibinger and Reiß [4]. Note the contrast with i.i.d. observations of a Gaussian vector where the empirical variance of one component is an efficient estimator and using the other entries cannot improve the variance estimator unless the correlation is known, cf. the classical Example 6.6.4 in Lehmann and Casella [18].

In Theorem 3.2 a multivariate central limit theorem (CLT) is provided for an oracle LMM (Local Method of Moments) estimator, using the unknown optimal weights and an information-type matrix for normalisation. Specifying to sample sizes of the same order $n$, Corollary (3.3) yields a CLT with rate $n^{1 / 4}$ and a covariance structure between matrix entries, which is given explicitly by concise matrix algebra. Using pre-estimated weight matrices, a 
fully adaptive version of the LMM-estimator is obtained, which by Theorem 3.4 shares the same asymptotic properties as the oracle estimator.

Another main result of this work is that the asymptotic covariance structure of the LMM-estimators is optimal in a semiparametric Cramér-Rao sense. In Section 4 a lower bound proof is achieved by a combination of space-time transformations and advanced calculus for covariance operators. The concrete form of the asymptotic variance is discussed for some key settings, thus generalising the univariate optimality theory by Gloter and Jacod [10] and Reiß [19] to the multivariate case. The discretisation and implementation of the estimator for model $\left(\mathcal{E}_{0}\right)$ is briefly described in Section 5 and presented together with some numerical results for a simple toy model and a more complex and realistic scenario. The finite sample behaviour of the LMM estimators is well predicted by the asymptotic theory (even in cases where it does not apply formally) and some comparison with competing procedures is provided.

\section{From discrete to continuous-time observations.}

2.1. Setting. First, let us specify different regularity assumptions. For functions $f:[0,1] \rightarrow \mathbb{R}^{m}, m \geq 1$ or also $m=d \times d$ for matrix values, we introduce the $L^{2}$-Sobolev ball of order $\alpha \in(0,1]$ and radius $R>0$

$$
H^{\alpha}(R)=\left\{f \in H^{\alpha}\left([0,1], \mathbb{R}^{m}\right) \mid\|f\|_{H^{\alpha}} \leq R\right\} \text { where }\|f\|_{H^{\alpha}}:=\max _{1 \leq i \leq m}\left\|f_{i}\right\|_{H^{\alpha}},
$$

which for matrices means $\|f\|_{H^{\alpha}}:=\max _{1 \leq i, j \leq d}\left\|f_{i j}\right\|_{H^{\alpha}}$. We also consider Hölder spaces $C^{\alpha}([0,1])$ and Besov spaces $B_{p, q}^{\alpha}([0,1])$ of such functions. Canonically, for matrices we use the spectral norm $\|\cdot\|$ and we set $\|f\|_{\infty}:=\sup _{t \in[0,1]}\|f(t)\|$.

In order to pursue asymptotic theory, we impose that the deterministic samplings in each component can be transferred to an equidistant scheme by respective quantile transformations independent of $n_{l}, 1 \leq l \leq d$.

Assumption 2.1.- $(\alpha)$ Suppose that there exist differentiable distribution functions $F_{l} \in C^{\alpha}([0,1]), 1 \leq l \leq d$, with $F_{l}(0)=0, F_{l}(1)=1$ and $F_{l}^{\prime}>0$, such that the observation times in $\left(\mathcal{E}_{0}\right)$ are generated by $t_{i}^{(l)}=F_{l}^{-1}\left(i / n_{l}\right)$, $0 \leq i \leq n_{l}, 1 \leq l \leq d$.

We gather all assertions on the instantaneous covolatility matrix function $\Sigma(t), t \in[0,1]$, which we shall require at some point. 
Assumption 2.2. Let $\Sigma:[0,1] \rightarrow \mathbb{R}^{d \times d}$ be a possibly random function with values in the class of symmetric, positive semi-definite matrices, independent of $X$ and the observational noise, satisfying:

(i- $\beta) \Sigma \in H^{\beta}([0,1])$ for $\beta>0$.

(ii- $\alpha) \Sigma=\Sigma^{B}+\Sigma^{M}$ with $\Sigma^{B} \in B_{1, \infty}^{\alpha}([0,1])$ for $\alpha>0$ and $\Sigma^{M}$ a matrixvalued $L^{2}$-martingale.

(iii- $\underline{\Sigma}) \Sigma(t) \geq \underline{\Sigma}$ for a strictly positive definite matrix $\underline{\Sigma}$ and all $t \in[0,1]$.

Let us briefly discuss the different function spaces, see e.g. Cohen [7, Section 3.2] for a survey. First, any $\alpha$-Hölder-continuous function lies in the $L^{2}$-Sobolev space $H^{\alpha}$ and any $H^{\alpha}$-function lies in the Besov space $B_{1, \infty}^{\alpha}$, where differentiability is measured in an $L^{1}$-sense. The important class of bounded variation functions (e.g., modeling jumps in the volatility) lies in $B_{1, \infty}^{1}$, but only in $H^{\alpha}$ for $\alpha<1 / 2$. In particular, part (ii- $\alpha$ ), $\alpha \leq 1$, covers $L^{2}$-semi-martingales by separate bounds on the drift (bounded variation) and martingale part. Beyond classical theory in this area is the fact that also non-semi-martingales like fractional Brownian motion $B^{H}$ with hurst parameter $H>1 / 2$ give rise to feasible volatility functions in the results below, using $B^{H} \in C^{H-\varepsilon} \cap B_{1, \infty}^{H}$ for any $\varepsilon>0$ from Ciesielski et al. [6].

In the sequel the potential randomness of $\Sigma$ is often not discussed additionally because by independence we can always work conditionally on $\Sigma$. Finally, let us mention that we could also weaken the Hölder-assumptions on $F_{1}, \ldots, F_{d}$ towards Sobolev or Besov regularity at the cost of tightening the assumptions on $\Sigma$. For the sake of clarity this is not pursued here.

Throughout the article we write $Z_{n}=\mathcal{O}_{P}\left(\delta_{n}\right)$ and $Z_{n}=\mathcal{O}_{P}\left(\delta_{n}\right)$ for a sequence of random variables $Z_{n}$ and a sequence $\delta_{n}$, to express that $\delta_{n}^{-1} Z_{n}$ is bounded or tends to zero in probability, respectively. Analogously $\mathcal{O}$ (or equivalently $\lesssim$ ) and $\mathcal{O}$ refer to deterministic sequences. We write $Z_{n} \asymp X_{n}$ if $Z_{n}=\mathcal{O}_{P}\left(Y_{n}\right)$ and $Y_{n}=\mathcal{O}_{P}\left(Z_{n}\right)$ and the same for deterministic quantities. $E_{d}$ denotes the $d$-dimensional unit matrix and $\delta_{p, q}=\mathbb{1}(p=q)$ equals 1 for $p=q$ and 0 otherwise.

\subsection{Continuous-time experiment.}

Definition 2.3. Let $\mathcal{E}_{0}\left(\left(n_{l}\right)_{1 \leq l \leq d}, \beta, R\right)$ with $n_{l} \in \mathbb{N}, \beta \in(0,1], R>$ 0 , be the statistical experiment generated by observations from $\left(\mathcal{E}_{0}\right)$ with $\Sigma \in H^{\beta}(R)$. Analogously, let $\mathcal{E}_{1}\left(\left(n_{l}\right)_{1 \leq l \leq d}, \beta, R\right)$ be the statistical experiment generated by observing $\left(\mathcal{E}_{1}\right)$ with the same parameter class.

As we shall establish next, experiments $\mathcal{E}_{0}$ and $\mathcal{E}_{1}$ will be asymptotically 
equivalent as $n_{l} \rightarrow \infty, 1 \leq l \leq d$, at a comparable speed, denoting

$$
n_{\min }=\min _{1 \leq l \leq d} n_{l} \text { and } n_{\max }=\max _{1 \leq l \leq d} n_{l} .
$$

Theorem 2.4. Grant Assumption 2.1-( $\beta)$ on the design. The statistical experiments $\mathcal{E}_{0}\left(\left(n_{l}\right)_{1 \leq l \leq d}, \beta, R\right)$ and $\mathcal{E}_{1}\left(\left(n_{l}\right)_{1 \leq l \leq d}, \beta, R\right)$ are asymptotically equivalent for any $\beta \in(0,1 / 2]$ and $R>0$, provided

$$
n_{\min } \rightarrow \infty, \quad n_{\max }=\mathcal{O}\left(\left(n_{\min }\right)^{1+\beta}\right) .
$$

More precisely, the Le Cam distance $\Delta$ is of order

$$
\Delta\left(\mathcal{E}_{0}\left(\left(n_{l}\right)_{1 \leq l \leq d}, \beta, R\right), \mathcal{E}_{1}\left(\left(n_{l}\right)_{1 \leq l \leq d}, \beta, R\right)\right)=\mathcal{O}\left(R^{2}\left(\sum_{l=1}^{d} n_{l} / \eta_{l}^{2}\right) n_{\text {min }}^{-1-\beta}\right) .
$$

By inclusion, the result also applies for $\beta>1 / 2$ when in the remaining expressions $\beta$ is replaced by $\min (\beta, 1 / 2)$. A standard Sobolev smoothness of $\Sigma$ is $\beta$ almost $1 / 2$ for diffusions with finitely many or absolutely summable jumps. In that case, the asymptotic equivalence result holds if $n_{\text {max }}$ grows more slowly than $n_{\text {min }}^{3 / 2}$. Theorem 2.4 is proved in the appendix in a constructive way by warped linear interpolation, which yields a readily implementable procedure, cf. Section 5 below.

\section{Localisation and method of moments.}

3.1. Construction. We partition the interval $[0,1]$ in blocks $[k h,(k+1) h)$ of length $h$. On each block a parametric MLE for a constant model could be sought for. Its numerical determination, however, is difficult and unstable due to the non-concavity of the ML objective function and its analysis is quite involved. Yet, the likelihood equation leads to spectral statistics whose empirical covariances estimate the quadratic covariation matrix. We therefore prefer a localised method of moments (LMM) for these spectral statistics where for an adaptive version the theoretically optimal weights are determined in a pre-estimation step, in analogy with the classical (multi-step) GMM (generalised method of moments) approach by Hansen [11].

As motivated in Reiß [19], consider the $L^{2}([0,1])$-orthonormal system $\left(\varphi_{j k}\right)$ and its antiderivatives $\left(\Phi_{j k}\right)$ :

$$
\begin{aligned}
\varphi_{j k}(t) & =\sqrt{\frac{2}{h}} \cos \left(j \pi h^{-1}(t-k h)\right) \mathbb{1}_{[k h,(k+1) h]}(t), j \geq 1, \\
\Phi_{j k}(t) & =\frac{\sqrt{2 h}}{j \pi} \sin \left(j \pi h^{-1}(t-k h)\right) \mathbb{1}_{[k h,(k+1) h]}(t), j \geq 1 .
\end{aligned}
$$


In each component local spectral statistics are defined by

$$
S_{j k}^{(l)}=\pi j h^{-1} \int_{k h}^{(k+1) h} \varphi_{j k}(t) d Y^{(l)}(t), j \geq 1, k=0, \ldots, h^{-1}-1,1 \leq l \leq d,
$$

from the continuous-time experiment $\mathcal{E}_{1}$. In order to design our estimator, we consider a locally constant approximation of the general non-parametric model.

Definition 3.1. Set $\bar{f}_{h}(t):=h^{-1} \int_{k h}^{(k+1) h} f(s) d s$ for $t \in[k h,(k+1) h)$, $k \in \mathbb{N}_{0}$, a function $f$ on $[0,1]$ and $h \in(0,1)$. Assume $h^{-1} \in \mathbb{N}$ and let $X_{t}^{h}=X_{0}+\int_{0}^{t} \bar{\Sigma}_{h}^{1 / 2}(s) d B_{s}$ with a d-dimensional standard Brownian motion $B$. Define the process

$$
d \tilde{Y}_{t}=X_{t}^{h} d t+\operatorname{diag}\left({\sqrt{\bar{H}^{2}}{ }_{n, l, h}(t)}_{1 \leq l \leq d} d W_{t}, t \in[0,1],\right.
$$

where $W$ is a standard Brownian motion independent of $B$ and with noise level (1.1). The statistical model generated by the observations from $\left(\mathcal{E}_{2}\right)$ for $\Sigma \in H^{\beta}(R)$ is denoted by $\mathcal{E}_{2}\left(\left(n_{l}\right)_{1 \leq l \leq d}, h, \beta, R\right)$.

In experiment $\mathcal{E}_{2}$ we thus observe a process with a covolatility matrix which is constant on each block $[k h,(k+1) h), k=0,1, \ldots, h^{-1}-1$, and corrupted by noise of block-wise constant magnitude. Our approach is founded on the idea that for small block sizes $h$ and sufficient regularity this piecewise constant approximation is close to $\mathcal{E}_{1}$.

A basic ingredient to derive the covariance structure are the joint moments for a $d$-dimensional random vector $X \sim \mathbf{N}(0, Q)$. For $(l, r, p, q) \in\{1, \ldots, d\}^{4}$ we have by Isserlis [13]:

$$
\mathbb{E}\left[X^{(l)} X^{(r)} X^{(p)} X^{(q)}\right]=Q_{l r} Q_{p q}+Q_{l p} Q_{r q}+Q_{l q} Q_{r p}
$$

in particular $\operatorname{Var}\left(\left(X^{(l)}\right)^{2}\right)=2 Q_{l l}^{2}, \operatorname{Var}\left(X^{(l)} X^{(p)}\right)=Q_{l l} Q_{p p}+Q_{l p}^{2}$.

The LMM estimator is built from the data in experiment $\mathcal{E}_{1}$, but designed for the block-wise parametric model $\mathcal{E}_{2}$. In $\mathcal{E}_{2}$, the $L^{2}$-orthogonality of $\left(\varphi_{j k}\right)$ as well as that of $\left(\Phi_{j k}\right)$ imply (cf. Reiß [19] in the scalar case)

$$
S_{j k} \sim \mathbf{N}\left(0, C_{j k}\right) \text { independent for all }(j, k)
$$

with covariance matrix

$$
C_{j k}=\Sigma^{k h}+\pi^{2} j^{2} h^{-2} \operatorname{diag}\left(H_{n, l}^{k h}\right)_{l}^{2}, \Sigma^{k h}=\bar{\Sigma}_{h}(k h), H_{n, l}^{k h}=\left({\overline{H^{2}}}_{n, l, h}(k h)\right)^{1 / 2} .
$$


In the multivariate central limit theorem we are facing covariances between entries of the covariation matrix estimator, which we shall formalise by interpreting matrices as vectors: for a matrix $A \in \mathbb{R}^{d \times d}$ we consider the vector of its entries

$$
\operatorname{vec}(A):=\left(A_{11}, A_{21}, \ldots, A_{d 1}, A_{12}, A_{22}, \ldots, A_{d 2}, \ldots, A_{d(d-1)}, A_{d d}\right)^{\top} \in \mathbb{R}^{d^{2}} .
$$

The natural estimator for $\operatorname{vec}\left(C_{j k}\right)$ is the empirical covariance $\operatorname{vec}\left(S_{j k} S_{j k}^{\top}\right)$. We employ Kronecker (tensor) product calculus, where $A \otimes B \in \mathbb{R}^{d^{2} \times d^{2}}$ for $A, B \in \mathbb{R}^{d \times d}$ is given by

$$
(A \otimes B)_{p(d-1)+q, p^{\prime}(d-1)+q^{\prime}}=A_{p p^{\prime}} B_{q q^{\prime}}, \quad p, q, p^{\prime}, q^{\prime}=1, \ldots, d .
$$

We evaluate the covariance matrix of $\operatorname{vec}\left(S_{j k} S_{j k}^{\top}\right)$ in model $\mathcal{E}_{2}$ by introducing

$$
\mathcal{Z}=\mathbb{C O V}\left(\operatorname{vec}\left(Z Z^{\top}\right)\right) \text { for } Z \sim \mathbf{N}\left(0, E_{d}\right)
$$

and applying the rule $\operatorname{vec}(A B C)=\left(C^{\top} \otimes A\right) \operatorname{vec}(B)$, see e.g. Fackler [9]:

$$
\operatorname{COV}_{\mathcal{E}_{2}}\left(\operatorname{vec}\left(S_{j k} S_{j k}^{\top}\right)\right)=\mathbb{C O V}\left(\operatorname{vec}\left(C_{j k}^{1 / 2} Z Z^{\top} C_{j k}^{1 / 2}\right)\right)=\left(C_{j k} \otimes C_{j k}\right) \mathcal{Z} .
$$

We have used the commutativity of $\mathcal{Z}$ with $\left(C_{j k} \otimes C_{j k}\right)^{1 / 2}=\left(C_{j k}^{1 / 2} \otimes C_{j k}^{1 / 2}\right)$, which is easily checked using the actual form of $\mathcal{Z}$ derived from (3.3)

$$
\mathcal{Z}_{p(d-1)+q, p^{\prime}(d-1)+q^{\prime}}=\left(1+\delta_{p, q}\right) \delta_{\{p, q\},\left\{p^{\prime}, q^{\prime}\right\}}, p, q, p^{\prime}, q^{\prime}=1, \ldots, d,
$$

or the equivalent property $\mathcal{Z} \operatorname{vec}(A)=\operatorname{vec}\left(A+A^{\top}\right)$ for all $A \in \mathbb{R}^{d \times d}$. Let us further introduce the Fisher information-type matrices

$$
I_{j k}=C_{j k}^{-1} \otimes C_{j k}^{-1}, \quad I_{k}=\sum_{j=1}^{\infty} I_{j k}, \quad j \geq 1, k=0, \ldots, h^{-1}-1 .
$$

Our local method of moments estimator with oracle weights $\mathrm{LMM}_{o r}^{(n)}$ uses that on each block a natural second moment estimator of $\Sigma^{k h}$ is given as a convex combination of the bias-corrected empirical covariances:

$$
\operatorname{LMM}_{o r}^{(n)}:=\sum_{k=0}^{h^{-1}-1} h \sum_{j=1}^{\infty} W_{j k} v e c\left(S_{j k} S_{j k}^{\top}-\frac{\pi^{2} j^{2}}{h^{2}} \operatorname{diag}\left(\left(H_{n, l}^{k h}\right)^{2}\right)_{1 \leq l \leq d}\right)
$$

The optimal weight matrices $W_{j k}$ in the oracle case are obtained as

$$
W_{j k}:=I_{k}^{-1} I_{j k} \in \mathbb{R}^{d^{2} \times d^{2}} .
$$

Note that $C_{j k}, I_{j k}, I_{k}$ and $W_{j k}$ all depend on $\left(n_{l}\right)_{1 \leq l \leq d}$ and $h$, which is omitted in the notation. Finally, observe that (3.5) and $\sum_{j} W_{j k}=E_{d^{2}}$ imply that $\mathrm{LMM}_{o r}^{(n)}$ is unbiased under $\mathcal{E}_{2}$. 
3.2. Asymptotic properties of the estimators. We formulate the main result that the oracle estimator (3.8) and also a fully adaptive version for the integrated volatility matrix satisfy central limit theorems.

TheOrem 3.2. Let Assumptions 2.1-( $\alpha)$, 2.2(ii- $\alpha)$ and 2.2(iii- $\underline{-})$ with $\alpha>1 / 2$ hold true for observations from model $\mathcal{E}_{1}$. The oracle estimator (3.8) yields a consistent estimator for $\operatorname{vec}\left(\int_{0}^{1} \Sigma(s) d s\right)$ as $n_{\min } \rightarrow \infty$ and $h=h_{0} n_{\min }^{-1 / 2}$ with $h_{0} \rightarrow \infty$. Moreover, if $n_{\max }=\mathcal{O}\left(n_{\min }^{2 \alpha}\right)$ and $h=\mathcal{O}\left(n_{\max }^{-1 / 4}\right)$, then a multivariate central limit theorem holds:

$$
\mathbf{I}_{n}^{1 / 2}\left(\operatorname{LMM}_{\text {or }}^{(n)}-\operatorname{vec}\left(\int_{0}^{1} \Sigma(s) d s\right)\right) \stackrel{\mathcal{L}}{\longrightarrow} \mathbf{N}(0, \mathcal{Z}) \text { in } \mathcal{E}_{1}
$$

with $\mathcal{Z}$ from (3.6) and $\mathbf{I}_{n}^{-1}=\sum_{k=0}^{h^{-1}-1} h^{2} I_{k}^{-1}$.

We give a typical illustration with convergence rate $n_{\text {min }}^{1 / 4}$ and asymptotic covariance matrix.

Corollary 3.3. Under the assumptions of Theorem 3.2 suppose $n_{\text {min }} / n_{p} \rightarrow \nu_{p} \in(0,1]$ for $p=1, \ldots, d$ and introduce $\mathcal{H}(t)=$ $\operatorname{diag}\left(\eta_{p} \nu_{p}^{1 / 2} F_{p}^{\prime}(t)^{-1 / 2}\right)_{p} \in \mathbb{R}^{d \times d}$ and $\Sigma_{\mathcal{H}}^{1 / 2}:=\mathcal{H}\left(\mathcal{H}^{-1} \Sigma \mathcal{H}^{-1}\right)^{1 / 2} \mathcal{H}$. Then

$$
n_{\text {min }}^{1 / 4}\left(\operatorname{LMM}_{\text {or }}^{(n)}-\operatorname{vec}\left(\int_{0}^{1} \Sigma(s) d s\right)\right) \stackrel{\mathcal{L}}{\longrightarrow} \mathbf{N}\left(0, \mathbf{I}^{-1} \mathcal{Z}\right) \text { in } \mathcal{E}_{1}
$$

with

$$
\mathbf{I}^{-1}=2 \int_{0}^{1}\left(\Sigma \otimes \Sigma_{\mathcal{H}}^{1 / 2}+\Sigma_{\mathcal{H}}^{1 / 2} \otimes \Sigma\right)(t) d t
$$

In particular, the entries satisfy for $p, q=1, \ldots, d$

$$
\begin{gathered}
n_{\min }^{1 / 4}\left(\left(\mathrm{LMM}_{o r}^{(n)}\right)_{p(d-1)+q}-\int_{0}^{1} \Sigma_{p q}(s) d s\right) \stackrel{\mathcal{L}}{\longrightarrow} \\
\mathbf{N}\left(0,2\left(1+\delta_{p, q}\right) \int_{0}^{1}\left(\Sigma_{p p}\left(\Sigma_{\mathcal{H}}^{1 / 2}\right)_{q q}+\Sigma_{q q}\left(\Sigma_{\mathcal{H}}^{1 / 2}\right)_{p p}+2 \Sigma_{p q}\left(\Sigma_{\mathcal{H}}^{1 / 2}\right)_{p q}\right)(t) d t\right) .
\end{gathered}
$$

The variance (3.12) will coincide with the lower bound obtained in Section 4 below. The local noise level in $\mathcal{H}(t)$ depends on the observational noise level $\eta_{p}$ and the local sample size $\nu_{p}^{-1} F_{p}^{\prime}(t), p=1, \ldots, d$, after normalisation by $n_{\text {min }}$. It is easy to see that in the case $n_{\min } / n_{p} \rightarrow 0$ the asymptotic variance vanishes for all entries $(p, q), q=1, \ldots, d$. In general, also all other asymptotic variances are then reduced because the estimator profits from correlation. In Section 4.2 the effect of $\mathcal{H}$ on $\Sigma_{\mathcal{H}}$ is discussed and illustrated 
in Figure 1. We infer the structure of the asymptotic covariance matrix using block-wise diagonalisation in Appendix B.

For a feasible estimator the optimal weight matrices $W_{j k}=W_{j}\left(\Sigma^{k h}\right)$ and the information-type matrices $I_{j k}=I_{j}\left(\Sigma^{k h}\right)$ are estimated in a preliminary step from the same data. To reduce variability in the estimate, a coarser grid of $r^{-1}$ equidistant intervals, $r / h \in \mathbb{N}$ is employed for $\hat{W}_{j k}$. As derived in Bibinger and Reiß [4] for supremum norm loss and extended to $L^{1}$-loss and Besov regularity using the $L^{1}$-modulus of continuity as in the case of wavelet estimators (Cor. 3.3.1 in Cohen [7]), a preliminary estimator $\hat{\Sigma}(t)$ of the instantaneous covolatility matrix $\Sigma(t)$ exists with

$$
\|\hat{\Sigma}-\Sigma\|_{L^{1}}=\mathcal{O}_{P}\left(n_{\min }^{-\alpha /(4 \alpha+2)}\right)
$$

for $\Sigma \in B_{1, \infty}^{\alpha}([0,1])$. For $k$ with $k h \in[m r,(m+1) r)$ we set

$$
\hat{W}_{j k}=W_{j}\left(\hat{\Sigma}^{m r}\right), \hat{I}_{j k}=I_{j}\left(\hat{\Sigma}^{k h}\right) \text { with } \hat{\Sigma}^{m r}=\overline{\hat{\Sigma}}_{r}(m r), \hat{\Sigma}^{k h}=\overline{\hat{\Sigma}}_{h}(k h) .
$$

The quadratic covariation matrix estimator with adaptive weights is then given by

$$
\operatorname{LMM}_{a d}^{(n)}=\sum_{k=0}^{h^{-1}-1} h \sum_{j=1}^{\infty} \hat{W}_{j k} \operatorname{vec}\left(S_{j k} S_{j k}^{\top}-\frac{\pi^{2} j^{2}}{h^{2}} \operatorname{diag}\left(\left(H_{n, l}^{k h}\right)^{2}\right)_{1 \leq l \leq d}\right)
$$

We estimate the total covariance matrix via

$$
\hat{\mathbf{I}}_{n}^{-1}=\sum_{k=0}^{h^{-1}-1} h^{2}\left(\sum_{j=1}^{\infty} \hat{I}_{j k}\right)^{-1}
$$

For $j \rightarrow \infty$ the weights $W_{j}(\Sigma)$ and the matrices $I_{j}(\Sigma)$ decay like $j^{-4}$ in norm, compare Lemma C.1 below, such that in practice a finite sum over frequencies $j$ suffices. By a tight bound on the derivatives of $\Sigma \mapsto W_{j}(\Sigma)$ we show in Appendix C.4 the following general result.

Theorem 3.4. Suppose $\Sigma \in B_{1, \infty}^{\alpha}([0,1])$ for $\alpha \in(1 / 2,1]$ satisfying $\alpha /(2 \alpha+1)>\log \left(n_{\max }\right) / \log \left(n_{\min }\right)-1$. Choose $h, r \rightarrow 0$ such that $h_{0}=$ $h n_{\text {min }}^{1 / 2} \asymp \log \left(n_{\text {min }}\right)$ and $n_{\text {min }}^{-\alpha /(2 \alpha+1)} \lesssim r \lesssim\left(n_{\text {min }} / n_{\text {max }}\right)^{1 / 2}, h^{-1}, r^{-1}, r / h \in$ $\mathbb{N}$. If the pilot estimator $\hat{\Sigma}$ satisfies (3.13), then under the conditions of Theorem 3.2 the adaptive estimator (3.14) satisfies

$$
\hat{\mathbf{I}}_{n}^{1 / 2}\left(\operatorname{LMM}_{a d}^{(n)}-\operatorname{vec}\left(\int_{0}^{1} \Sigma(s) d s\right)\right) \stackrel{\mathcal{L}}{\longrightarrow} \mathbf{N}(0, \mathcal{Z}),
$$

with $\hat{\mathbf{I}}_{n}$ from (3.15).

Moreover, Corollary 3.3 applies equally to the adaptive estimator (3.14). 
Since the estimated $\hat{\mathbf{I}}_{n}$ appears in the CLT, we have obtained a feasible limit theorem and (asymptotic) inference statements are immediate.

Some assumptions of Theorem 3.4 are tighter than for the oracle estimator. To some extent this is for the sake of clarity. Here, we have restricted Assumption 2.2(ii- $\alpha)$ to the Besov-regular part. A generalisation of the pilot estimator to martingales seems feasible, but is non-standard and might require additional conditions. We have also proposed a rather concrete choice of $h$ and $r$, less is used in the proof, see e.g. (C.3) below.

The lower bound for $\alpha$ in terms of the sample-size ratio $n_{\max } / n_{\min }$ is due to bounding norms of (estimated) information-type matrices separately. For $\alpha=1$ (bounded variation case) the restriction imposes $n_{\max }$ to be somewhat smaller than $n_{\text {min }}^{4 / 3}$. By the Sobolev embedding $B_{1, \infty}^{1} \subseteq H^{\beta}$ for all $\beta<1 / 2$ the restriction $n_{\max }=\mathcal{O}\left(n_{\min }^{1+\beta}\right)$ from Theorem 2.4 is clearly also satisfied in this case.

It is not clear whether a more elaborate analysis can avoid these restrictions. Still, to the best of our knowledge, a feasible CLT for asymptotically separating sample sizes has not been obtained before. The inclusion of possible jumps in $\Sigma$ by measuring regularity only in the Besov scale is already in the scalar case a significant improvement over Reiß [19].

\section{Semiparametric efficiency.}

4.1. Semiparametric Cramér-Rao bound. We shall derive an efficiency bound for the following basic case of observation model $\left(\mathcal{E}_{1}\right)$ :

$$
d Y_{t}=X_{t} d t+\frac{1}{\sqrt{n}} d W_{t}, \quad X_{t}=\int_{0}^{t} \Sigma(s)^{1 / 2} d B_{s}, \quad t \in[0,1]
$$

where

$$
\Sigma(t)=\Sigma_{0}(t)+\varepsilon \mathbb{H}(t), \quad \Sigma_{0}(t)^{1 / 2}=O(t)^{\top} \Lambda(t) O(t) .
$$

We assume $\Sigma_{0}(t)$ and $\mathbb{H}(t)$ to be known symmetric matrices, $O(t)$ orthogonal matrices, $\Lambda(t)=\operatorname{diag}\left(\lambda_{1}(t), \ldots, \lambda_{d}(t)\right)$ diagonal and consider $\varepsilon \in[-1,1]$ as unknown parameter. Furthermore, we require Assumption 2.2(iii- $\underline{\Sigma})$ for all $\Sigma$. Finally, we impose throughout this section the regularity assumption that the matrix functions $O(t), \mathbb{H}(t), \Lambda(t)$ are continuously differentiable.

The key idea is to transform the observation of $d Y_{t}$ in such a manner that the white noise part remains invariant in law while for the central parameter $\Sigma(t)=\Sigma_{0}(t)$ the process $X$ is transformed to a process with independent coordinates and constant volatility. It turns out that this can only be achieved 
at the cost of an additional drift in the signal. The construction first rotates the observations via $O(t)$, which diagonalises $\Sigma_{0}(t)$, and then applies a coordinate-wise time-transformation, corrected by a multiplication term to ensure $L^{2}$-isometry such that the white noise remains law-invariant.

We introduce the coordinate-wise time changes by

$$
r_{i}(t)=\frac{\int_{0}^{t} \lambda_{i}(s) d s}{\int_{0}^{1} \lambda_{i}(s) d s} \text { and }\left(T_{r} g\right)(t):=\left(g_{1}\left(r_{1}(t)\right), \ldots, g_{d}\left(r_{d}(t)\right)\right)^{\top}
$$

for $g=\left(g_{1}, \ldots, g_{d}\right): \mathbb{R} \rightarrow \mathbb{R}^{d}$. Moreover, we set

$$
\bar{\Lambda}:=\int_{0}^{1} \Lambda(s) d s, \quad R^{\prime}(t):=\bar{\Lambda}^{-1} \Lambda(t)=\operatorname{diag}\left(r_{1}^{\prime}(t), \ldots, r_{d}^{\prime}(t)\right) .
$$

Lemma 4.1. By transforming $d \bar{Y}=T_{r}^{-1} \mathcal{M}_{\left(R^{\prime}\right)^{-1 / 2} O} d Y$, the observation model (4.1), (4.2) is equivalent to observing

$$
\begin{gathered}
d \bar{Y}(t)=S(t) d t+\frac{1}{\sqrt{n}} d \bar{W}(t) \quad \text { with } \\
S(t)=T_{r}^{-1}\left(\left(R^{\prime}\right)^{-1}\left(\int_{0}\left(\left(R^{\prime}\right)^{-\frac{1}{2}} O\right)^{\prime}(s) X(s) d s+\int_{0}\left(R^{\prime}(s)\right)^{-\frac{1}{2}} O(s) d X(s)\right)\right)(t)
\end{gathered}
$$

for $t \in[0,1]$. At $\varepsilon=0$ the observation $d \bar{Y}(t)$ reduces to

$$
\left(\int_{0}^{t} T_{r}^{-1}\left(\left(R^{\prime}\right)^{-1}\left(\left(R^{\prime}\right)^{-1 / 2} O\right)^{\prime} X\right)(s) d s+\bar{\Lambda} \bar{B}(t)\right) d t+\frac{1}{\sqrt{n}} d \bar{W}(t) .
$$

Here $\bar{W}$ and $\bar{B}$ are Brownian motions obtained from $W$ and B, respectively, via rotation and time shift, as defined in (D.1) below.

If we may forget in (4.4) the first term, which is a drift term with respect to the martingale part $\bar{\Lambda} \bar{B}(t)$, then the central observation is indeed a constant volatility model in white noise. The lemma is proved in Appendix D.1.

Let us introduce the multiplication operator $\mathcal{M}_{A} g:=A g$ and the integration operator

$$
I g(t)=-\int_{t}^{1} g(s) d s \text { and its adjoint } I^{*} g(t)=-\int_{0}^{t} g(s) d s .
$$

The covariance operator $C_{n, \varepsilon}$ on $L^{2}\left([0,1], \mathbb{R}^{d}\right)$ obtained from observing the differential in (4.3) is then given by

$$
C_{n, \varepsilon}=T_{r}^{*} \mathcal{M}_{\left(R^{\prime}\right)^{1 / 2} O} I^{*} \mathcal{M}_{\Sigma_{0}+\varepsilon \mathbb{H}} I \mathcal{M}_{O^{\top}\left(R^{\prime}\right)^{1 / 2}} T_{r}+n^{-1} \mathrm{Id}
$$


The covariance operator $Q_{n, \varepsilon}$ when omitting the drift part is given by

$$
Q_{n, \varepsilon}=Q_{n, 0}+\varepsilon I^{*} T_{r}^{*} \mathcal{M}_{M} T_{r} I \text { with } M(t):=\left(\left(R^{\prime}\right)^{-1 / 2} O H O^{\top}\left(R^{\prime}\right)^{-1 / 2}\right)(t)
$$

where for $\varepsilon=0$ the one-dimensional Brownian motion covariance operator $C_{B M}$ appears

$$
Q_{n, 0}=\operatorname{diag}\left(\bar{\lambda}_{i i} C_{B M}+n^{-1} \mathrm{Id}\right)_{1 \leq i \leq d}, \quad C_{B M}=I^{*} I .
$$

We set $\dot{C}_{0}=\left(C_{n, \varepsilon}-C_{n, 0}\right) / \varepsilon$ and $\dot{Q}_{0}=\left(Q_{n, \varepsilon}-Q_{n, 0}\right) / \varepsilon$.

Standard Fisher information calculations for the finite-dimensional Gaussian scale model, e.g. [18, Chapter 6.6], transfer one-to-one to the infinitedimensional case of observing $\mathbf{N}\left(0, Q_{n, \varepsilon}\right)$ and yield as Fisher information for the parameter $\varepsilon$ at $\varepsilon=0$ the value

$$
I_{n}^{Q}=\frac{1}{2}\left\|Q_{n, 0}^{-1 / 2} \dot{Q}_{0} Q_{n, 0}^{-1 / 2}\right\|_{H S}^{2},
$$

because $Q_{n, 0}^{-1 / 2} Q_{n, \varepsilon} Q_{n, 0}^{-1 / 2}$ is differentiable at $\varepsilon=0$ in Hilbert-Schmidt norm. In Appendix D.2 we show by Hilbert-Schmidt calculus, the Feldman-Hajek Theorem and the Girsanov Theorem that the models with and without drift do not separate:

LEMma 4.2. We have

$$
\limsup _{n \rightarrow \infty}\left\|Q_{n, 0}^{-1 / 2} \dot{Q}_{0} Q_{n, 0}^{-1 / 2}-C_{n, 0}^{-1 / 2} \dot{C}_{0} C_{n, 0}^{-1 / 2}\right\|_{H S}<\infty .
$$

Lemma 4.2 implies that the drift only contributes the negligible order $\mathcal{O}(1)=\mathcal{O}(\sqrt{n})$ to the Fisher information. By identifying the hardest parametric subproblem for observations $\mathbf{N}\left(0, Q_{n, \varepsilon}\right)$ we thus establish in Appendix D.3 a semiparametric Cramér-Rao bound for estimating any linear functional of the covolatility matrix. Further classical asymptotic statements like the local asymptotic minimax theorem would require the LAN-property of the parametric subproblem.

Theorem 4.3. For a continuous matrix-valued function $A:[0,1] \rightarrow$ $\mathbb{R}^{d \times d}$ consider the estimation of

$$
\vartheta:=\int_{0}^{1}\langle A(t), \Sigma(t)\rangle_{H S} d t=\int_{0}^{1} \sum_{i, j=1}^{d} A_{i j}(t) \Sigma_{i j}(t) d t \in \mathbb{R} .
$$

Then a hardest parametric subproblem in model (4.1), (4.2) is obtained for the perturbation of $\Sigma_{0}$ by

$$
\mathbb{H}^{*}(t)=\left(\Sigma_{0}\left(A+A^{\top}\right) \Sigma_{0}^{1 / 2}+\Sigma_{0}^{1 / 2}\left(A+A^{\top}\right) \Sigma_{0}\right)(t) .
$$


There any estimator $\hat{\vartheta}_{n}$ of $\vartheta$, which is asymptotically unbiased in the sense $\frac{d}{d \vartheta}\left(\mathbb{E}_{\vartheta}\left[\hat{\vartheta}_{n}\right]-\vartheta\right) \rightarrow 0$, satisfies as $n \rightarrow \infty$

$\operatorname{Var}_{\varepsilon=0}\left(\hat{\vartheta}_{n}\right) \geq \frac{(2+\mathcal{O}(1))}{\sqrt{n}} \int_{0}^{1}\left\langle\left(\Sigma_{0} \otimes \Sigma_{0}^{1 / 2}+\Sigma_{0}^{1 / 2} \otimes \Sigma_{0}\right) \mathcal{Z} \operatorname{vec}(A), \mathcal{Z} v e c(A)\right\rangle(t) d t$.

4.2. Discussion. The Cramér-Rao bound of Theorem 4.3 coincides with the asymptotic variance obtained in Corollary 3.3 in the case $\mathcal{H}(t)=E_{d}$. For different, but constant in time noise levels $\mathcal{H}$, we can apply a rescaling argument and replace in the lower bound model $\Sigma(t)$ by $\mathcal{H}^{-1} \Sigma(t) \mathcal{H}^{-1}$ and $A(t)$ by $\mathcal{H} A(t) \mathcal{H}$. This gives the more general Cramér-Rao bound

$\operatorname{Var}_{\varepsilon=0}\left(\hat{\vartheta}_{n}\right) \geq \frac{(2+\mathcal{O}(1))}{\sqrt{n}} \int_{0}^{1}\left\langle\left(\Sigma_{0} \otimes \Sigma_{0, \mathcal{H}}^{1 / 2}+\Sigma_{0, \mathcal{H}}^{1 / 2} \otimes \Sigma_{0}\right) \mathcal{Z} v e c(A), \mathcal{Z} v e c(A)\right\rangle(t) d t$

with $\Sigma_{0, \mathcal{H}}^{1 / 2}=\mathcal{H}\left(\mathcal{H}^{-1} \Sigma_{0} \mathcal{H}^{-1}\right)^{1 / 2} \mathcal{H}$, as in Corollary 3.3. If $\mathcal{H}(t)$ depends on $t$, rescaling generates another drift term, but if it varies smoothly in $t$, we expect to obtain again a lower bound that matches the asymptotic variance of our estimator. Let us discuss the efficient asymptotic variance AVAR further, concentrating on the homogeneous case $\mathcal{H}(t)=E_{d}$.

The efficient asymptotic variance for estimating $\int_{0}^{1} \Sigma_{p p}(t) d t$ is

$$
\operatorname{AVAR}\left(\int_{0}^{1} \Sigma_{p p}(t) d t\right)=8 \int_{0}^{1} \Sigma_{p p}(t)\left(\Sigma^{1 / 2}(t)\right)_{p p} d t
$$

For the asymptotic variance of estimating $\int_{0}^{1} \Sigma_{p q}(t) d t$ we obtain

$$
2 \int_{0}^{1}\left(\left(\Sigma^{1 / 2}\right)_{p p} \Sigma_{q q}+\left(\Sigma^{1 / 2}\right)_{q q} \Sigma_{p p}+2\left(\Sigma^{1 / 2}\right)_{p q} \Sigma_{p q}\right)(t) d t .
$$

Let us calculate specific examples. First, in the case $d=1, \Sigma=\sigma^{2}$ this simplifies to

$$
\operatorname{AVAR}\left(\int_{0}^{1} \sigma^{2}(t) d t\right)=8 \int_{0}^{1} \sigma^{3}(t) d t
$$

which agrees with the efficiency bound in Reiß [19]. For $p \neq q$ in the independent case $\Sigma=\operatorname{diag}\left(\sigma_{p}^{2}\right)_{1 \leq p \leq d}$ we find

$$
\operatorname{AVAR}\left(\int_{0}^{1} \Sigma_{p q}(t) d t\right)=2 \int_{0}^{1}\left(\sigma_{p}^{2} \sigma_{q}+\sigma_{p} \sigma_{q}^{2}\right)(t) d t
$$

In the case $d=2$ with spot volatilities $\sigma_{1}^{2}(t)=\sigma_{2}^{2}(t)=\sigma^{2}(t)$ and general correlation $\rho(t)$, i.e. $\sigma_{12}(t)=\left(\rho \sigma_{1} \sigma_{2}\right)(t)$, we obtain

$$
\begin{aligned}
& \operatorname{AVAR}\left(\int_{0}^{1} \sigma_{1}^{2}(t) d t\right)=4 \int_{0}^{1} \sigma^{3}(t)(\sqrt{1+\rho(t)}+\sqrt{1-\rho(t)}) d t \\
& \operatorname{AVAR}\left(\int_{0}^{1} \sigma_{12}(t) d t\right)=2 \int_{0}^{1} \sigma^{3}(t)\left((1+\rho(t))^{3 / 2}+(1-\rho(t))^{3 / 2}\right) d t
\end{aligned}
$$



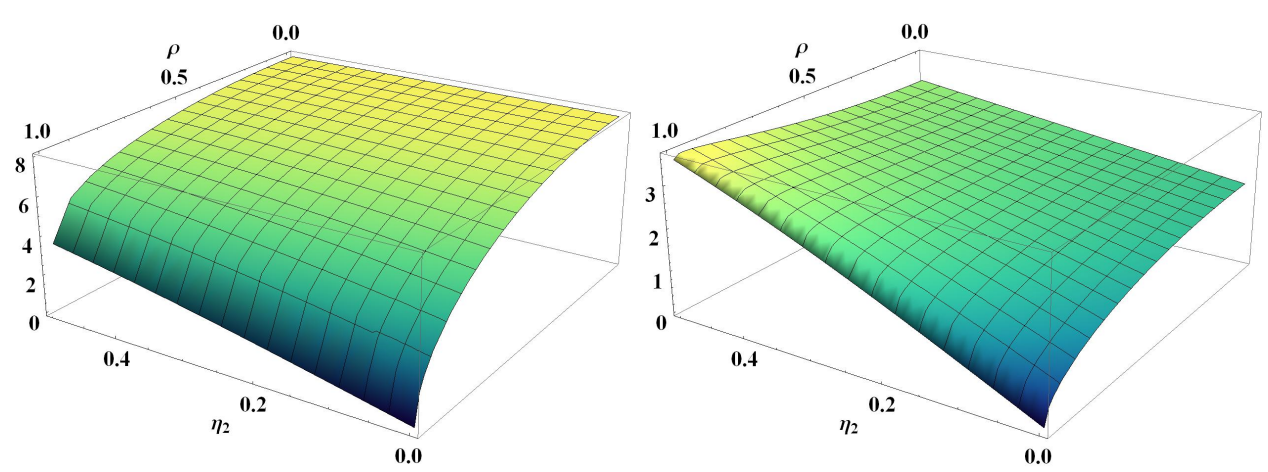

FIG 1. Asymptotic variances of LMM for volatility $\sigma_{1}^{2}$ (left) and covolatility $\sigma_{12}$ (right) plotted against correlation $\rho$ and noise level $\eta_{2}$ (constant in time).

With time-constant parameters these bounds decay for $\sigma_{1}^{2}$ (resp. grow for $\sigma_{12}$ ) in $|\rho|$ from $8 \sigma^{3}$ (resp. $4 \sigma^{3}$ ) at $\rho=0$ to $4 \sqrt{2} \sigma^{3}$ at $|\rho|=1$ for both cases.

All the preceding examples can be worked out for different noise levels in $\mathcal{H}$, let us just highlight the bound for $\operatorname{AVAR}\left(\int_{0}^{1} \Sigma_{p q}(t) d t\right)$ :

$$
2 \int_{0}^{1}\left(\left(\Sigma_{\mathcal{H}}^{1 / 2}\right)_{p p} \Sigma_{q q}+\left(\Sigma_{\mathcal{H}}^{1 / 2}\right)_{q q} \Sigma_{p p}+2\left(\Sigma_{\mathcal{H}}^{1 / 2}\right)_{p q} \Sigma_{p q}\right)(t) d t
$$

which matches the asymptotic variances obtained in Corollary 3.3. In general all noise levels enter for a fixed entry $(p, q)$ via the matrix $\operatorname{root}\left(\mathcal{H}^{-1} \Sigma \mathcal{H}^{-1}\right)^{1 / 2}$ which only in the case of a diagonal covolatility matrix $\Sigma=\operatorname{diag}\left(\sigma_{p}^{2}\right)_{p}$ decouples as $\operatorname{diag}\left(\eta_{p}^{-1} \sigma_{p}\right)_{p}$ and where the bound simplifies to

$$
p \neq q: 2 \int_{0}^{1}\left(\eta_{p} \sigma_{p} \sigma_{q}^{2}+\eta_{q} \sigma_{q} \sigma_{p}^{2}\right)(t) d t ; \quad p=q: 8 \int_{0}^{1}\left(\eta_{p} \sigma_{p}^{3}\right)(t) d t .
$$

Figure 1 illustrates the general dependence of the asymptotic variance on the noise level via $\Sigma_{\mathcal{H}}$ in the case $d=2$. The two volatilities are $\sigma_{1}=\sigma_{2}=1$, the covolatility is $\sigma_{12}=\rho$ (constant in time) and the first noise level is $\eta_{1}=1$. The left plot shows the asymptotic variance of the estimator of $\sigma_{1}^{2}$ as a function of $\rho$ and $\eta_{2}$. We see the significant gain of using observations from the other process for larger values of $\rho$. If the noise level $\eta_{2}$ for the second process is small, then the asymptotic variance can even approach zero. The plot on the right shows the same dependence for estimating the covolatility $\sigma_{12}$. For comparable size of $\eta_{2}$ and $\eta_{1}$ the asymptotic variance increases in $\rho$ which is explained by the fact that also the value to be estimated increases. For small values of $\eta_{2}$, however, the efficiency gain by exploiting the correlation prevails. 
For larger dimension $d$ the variance can even be of order $\mathcal{O}(1 / \sqrt{d})$; in the concrete case where all volatilities and noise levels equal 1, the asymptotic variance for estimating $\sigma_{1}^{2}$ can be reduced from 8 (using only observations from the first component or if $\Sigma$ is diagonal) down to $8 / \sqrt{d}$ (in case of perfect correlation).

We can also investigate the estimation of the entire quadratic covariation matrix $\int_{0}^{1} \Sigma(t) d t$ and measure its loss with respect to the squared $d \times d$ Hilbert-Schmidt norm. Summing up the variances for each entry, we obtain the asymptotic risk

$$
\frac{4}{\sqrt{n}} \int_{0}^{1}\left(\operatorname{trace}\left(\Sigma^{1 / 2}\right) \operatorname{trace}(\Sigma)+\operatorname{trace}\left(\Sigma^{3 / 2}\right)\right)(t) d t .
$$

This can be compared with the corresponding Hilbert-Schmidt norm error $\frac{1}{n}\left(\operatorname{trace}(\Sigma)^{2}+\operatorname{trace}\left(\Sigma^{2}\right)\right)$ for the empirical covariance matrix in an i.i.d. Gaussian $\mathbf{N}(0, \Sigma)$-setting.

For nonlinear functionals the Cramér-Rao bound is obtained by linearisation. Consider the prominent example of estimating power variations of the form $\int_{0}^{1}\left(\Sigma_{p q}\right)^{\rho / 2}(t) d t$ for some $\rho>0(\rho=4$ yields the socalled quarticity). Linearisation of the perturbation yields $\Sigma_{p q}^{\rho / 2}=\left(\Sigma_{0}\right)_{p q}^{\rho / 2}+$ $\frac{\rho}{2}\left(\Sigma_{0}\right)_{p q}^{\rho / 2-1} \varepsilon H_{p q}+\mathcal{O}(\varepsilon)$ provided $\left(\Sigma_{0}\right)_{p q}>0$. We thus consider $A(t)=$ $\frac{\rho}{2}\left(\Sigma_{0}(t)\right)_{p q}^{\rho / 2-1}\left(\delta_{(p, q),\left(p^{\prime}, q^{\prime}\right)}\right)_{p^{\prime}, q^{\prime}}$ and obtain the lower bound

$$
\frac{\rho^{2}}{2} \int_{0}^{1}\left(\Sigma_{0}\right)_{p q}^{\rho-2}\left(\left(\Sigma_{0}^{1 / 2}\right)_{p p}\left(\Sigma_{0}\right)_{q q}+\left(\Sigma_{0}^{1 / 2}\right)_{q q}\left(\Sigma_{0}\right)_{p p}+2\left(\Sigma_{0}^{1 / 2}\right)_{p q}\left(\Sigma_{0}\right)_{p q}\right)(t) d t
$$

For $p=q$ this reduces to $2 \rho^{2} \int_{0}^{1}\left(\left(\Sigma_{0}^{1 / 2}\right)_{p p}\left(\Sigma_{0}\right)_{p p}^{\rho-1}\right)(t) d t$, which is independent of $\Sigma_{0}$ only if $\rho=1 / 2$ and $\Sigma_{0}$ is diagonal. In that case asymptotic equivalence with a homoskedastic Gaussian shift in terms of the mean function $\Sigma(t)^{1 / 4}$ holds, derived by independence of coordinates from Reiß [19], but for nondiagonal $\Sigma_{0}$ a variance-stabilising transform or even an equivalence result is not apparent.

\section{Implementation and numerical results.}

5.1. Discrete-time estimator. The construction to transfer discrete-time to continuous-time observations in the proof of Theorem 2.4 paves the way to the discrete approximation of the local spectral statistics (3.2). Using the interpolated process and integration by parts yields

$$
\int \varphi_{j k}(t) d Y^{(l)}(t) \asymp-\sum_{\nu=1}^{n_{l}} \int_{t_{\nu-1}^{(l)}}^{t_{\nu}^{(l)}} \Phi_{j k}(t) \frac{Y_{\nu}^{(l)}-Y_{\nu-1}^{(l)}}{t_{\nu}^{(l)}-t_{\nu-1}^{(l)}} d t .
$$



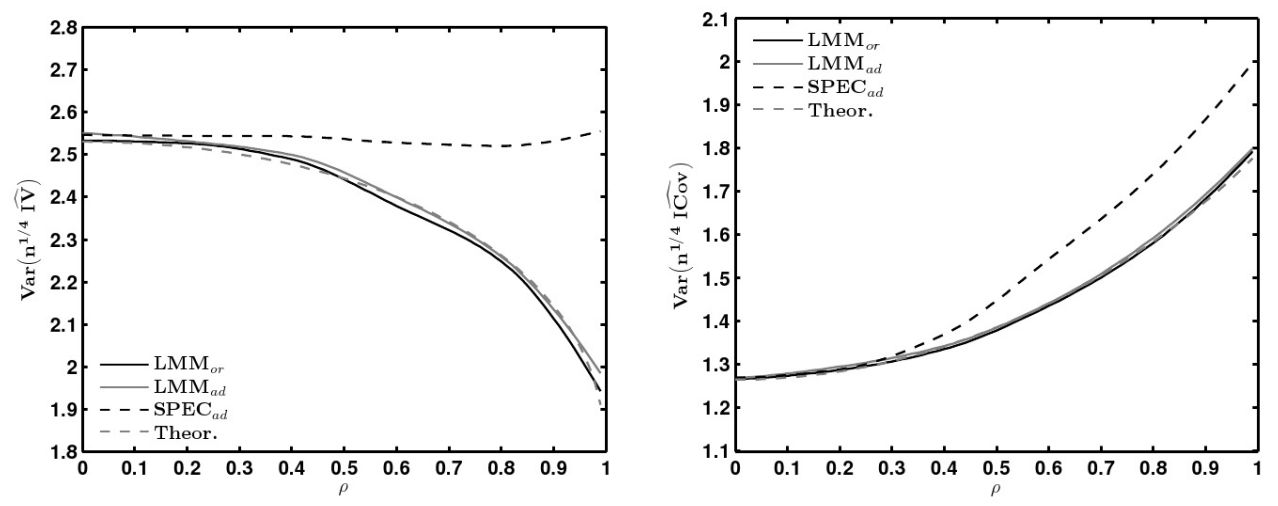

FIG 2. Variances of estimators of $\sigma_{1}^{2}$ (left) and $\sigma_{12}$ (right) in time-constant scenario $(n=30,000)$.

Hence, for discrete-time observations from $\left(\mathcal{E}_{0}\right)$ we use the local spectral statistics

$$
S_{j k}=\pi j h^{-1}\left(\sum_{\nu=1}^{n_{l}}\left(Y_{\nu}^{(l)}-Y_{\nu-1}^{(l)}\right) \Phi_{j k}\left(\frac{t_{\nu-1}^{(l)}+t_{\nu}^{(l)}}{2}\right)\right)_{1 \leq l \leq d} .
$$

The noise terms in (3.5) translate from $\mathcal{E}_{1}$ to $\mathcal{E}_{0}$ via substituting $n_{l}^{-1} \int_{k h}^{(k+1) h}\left(F_{l}^{\prime}(s)\right)^{-1} d s$ by $\sum_{\nu: k h \leq t_{\nu}^{(l)} \leq(k+1) h}\left(t_{\nu}^{(l)}-t_{\nu-1}^{(l)}\right)^{2}$. The discrete sum times $h^{-1}$ can be understood as a block-wise quadratic variation of time in the spirit of Zhang et al. [20]. The bias is discretised analogously.

For the adaptive estimator we are in need of local estimates of $n_{l} F_{l}^{\prime}, \Sigma$ and estimators for $\eta_{l}^{2}, 1 \leq l \leq d$. It is well known how to estimate noise variances with faster $\sqrt{n_{l}}$-rates, see e.g. Zhang et al. [20]. Local observation densities can be estimated with block-wise quadratic variation of time as above, which then yield estimators $\hat{H}_{n, l}^{k h}$ of $H_{n, l}$ around time $k h$. Uniformly consistent estimators for $\Sigma(t), t \in[0,1]$, are feasible, e.g. averaging spectral statistics for $j=1, \ldots, J$ over a set $\mathcal{K}_{t}$ of $K$ adjacent blocks containing $t$ :

$$
\widehat{\Sigma}(t)=K^{-1} \sum_{k \in \mathcal{K}_{t}} J^{-1} \sum_{j=1}^{J}\left(S_{j k} S_{j k}^{\top}-\pi^{2} j^{2} h^{-2} \operatorname{diag}\left(\left(\hat{H}_{n, l}^{k h}\right)_{l}^{2}\right)\right),
$$

We refer to Bibinger and Reiß [4] for details on the non-parametric pilot estimator with $J=1$.

5.2. Simulations. We examine the finite-sample properties of the LMM for the case $d=2$ in two scenarios. First, we compare the finite-sample vari- 
ance with the asymptotic variances from Sections 3 and 4, i.e.for a parametric setup with $\eta_{1}^{2}=\eta_{2}^{2}=0.1, \sigma_{1}=\sigma_{2}=1$ and constant correlation $\rho$. We simulate $n_{1}=n_{2}=30,000$ synchronous observations on $[0,1]$. For estimating $\sigma_{1}^{2}$ and $\sigma_{12}=\rho$, Figure 2 displays the rescaled Monte-Carlo variance based on 20,000 replications of the oracle and adaptive LMM $\left(\mathrm{LMM}_{o r}\right.$ and $\left.\mathrm{LMM}_{a d}\right)$, as well as the adaptive spectral estimator $\left(\mathrm{SPEC}_{a d}\right)$ by Bibinger and Reiß [4], which relies on the same spectral approach, but uses only scalar weighting instead of the full information matrix approach.

In practice the pilot estimator from (5.2) for $J$ not too large performed well. As configuration we use $h^{-1}=10, J=30$ and $K=8$, which turned out to be an accurate choice, but the estimators are reasonably robust to alternative input choices. For the LMM of $\sigma_{1}^{2}$, we observe the already familiar variance reduction effect associated with a growing signal correlation $\rho$, while the simulation-based variances of both $\mathrm{LMM}_{\text {or }}$ and $\mathrm{LMM}_{a d}$ are close to their theoretical asymptotic counterpart (Theor). The results for $\sigma_{12}$ underline the precision gains compared to $\mathrm{SPEC}_{a d}$ with univariate weights when $\rho$ increases.

Next, we consider a complex and realistic stochastic volatility setting that relies on an extension of the widely-used Heston model, as e. g. employed by Aït-Sahalia et al. [1], accounting for both leverage effects and an intraday seasonality of volatility. The signal process for $l=1,2$ evolves as

$$
d X_{t}^{(l)}=\varphi_{l}(t) \sigma_{l}(t) d Z_{t}^{(l)}, \quad d \sigma_{l}^{2}(t)=\alpha_{l}\left(\mu_{l}-\sigma_{l}^{2}(t)\right) d t+\psi_{l} \sigma_{l}(t) d V_{t}^{(l)},
$$

where $Z_{t}^{(l)}$ and $V_{t}^{(l)}$ are standard Brownian motions with $d Z_{t}^{(1)} d Z_{t}^{(2)}=\rho d t$ and $d Z_{t}^{(l)} d V_{t}^{(m)}=\delta_{l, m} \gamma_{l} d t . \varphi_{l}(t)$ is a non-stochastic seasonal factor with $\int_{0}^{1} \varphi_{l}^{2}(t) d t=1$. The unit time interval can represent one trading day, e.g. 6.5 hours or 23,400 seconds at NYSE.

We initialise the variance process $\sigma_{l}^{2}(t)$ by sampling from its stationary distribution $\Gamma\left(2 \alpha_{l} \mu_{l} / \psi_{l}^{2}, \psi_{l}^{2} /\left(2 \alpha_{l}\right)\right)$ and vary the value of the instantaneous signal correlation $\rho$, while setting $\left(\mu_{l}, \alpha_{l}, \psi_{l}, \gamma_{l}\right)=(1,6,0.3,-0.3), l=1,2$, which under the stationary distribution, implies $\mathbb{E}\left[\int_{0}^{1} \varphi_{l}^{2}(t) \sigma_{l}^{2}(t) d t\right]=1$. The seasonal factor $\varphi_{l}(t)$ is specified in terms of intraday volatility functions estimated for S\&P 500 equity data by the procedure in Anderson and Bollerslev [2]. $\varphi_{1}(t)$ and $\varphi_{2}(t)$ are based on cross-sectional averages of the 50 most and 50 least liquid stocks, respectively, which yields a pronounced L-shape in both cases (see Figure 3 ). We add noise processes that are i.i.d. $\mathbf{N}\left(0, \eta_{l}^{2}\right)$ and mutually independent with $\eta_{l}=0.1\left(\mathbb{E}\left[\int_{0}^{1} \varphi_{l}^{4}(t) \sigma_{l}^{4}(t) d t\right]\right)^{1 / 4}$, computed under the stationary distribution of $\sigma_{l}^{2}(t)$. Finally, asynchronicity effects are introduced by drawing observation times $t_{i}^{(l)}, 1 \leq i \leq n_{l}, l=1,2$, from two 

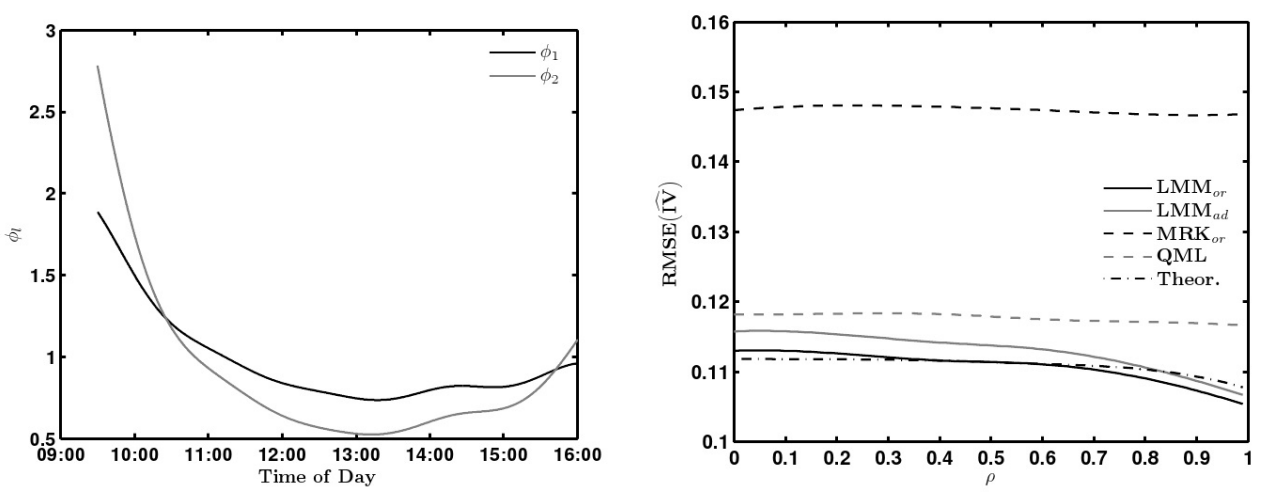

FIG 3. Non-stochastic volatility seasonality factors (left) and RMSE for estimators of $\int_{0}^{1} \varphi_{1}^{2}(t) \sigma_{1}^{2}(t) d t$ (right) in stochastic volatility scenario.

independent Poisson processes with intensities $\lambda_{1}=1$ and $\lambda_{2}=2 / 3$ such that, on average, $n_{1}=23,400$ and $n_{2}=15,600$.

As a representative example, Figure 3 depicts the root mean-squared errors (RMSEs) based on 40,000 replications of the following estimators of $\int_{0}^{1} \varphi_{1}^{2}(t) \sigma_{1}^{2}(t) d t$ : the oracle and adaptive LMM using $h^{-1}=20, J=15$ and $K=8$, the quasi-maximum likelihood (QML) estimator by Ait-Sahalia et. al. [1] as well as an oracle version of the widely-used multivariate realised kernel $\left(\mathrm{MRK}_{o r}\right)$ by Barndorff-Nielsen et al. [3]. For the latter, we employ the average univariate mean-squared error optimal bandwidth based on the true value of $\int_{0}^{1} \varphi_{l}^{4}(t) \sigma_{l}^{4}(t) d t, l=1,2$. Further, we include the theoretical variance from the asymptotic theory (Theor), which is computed as the variance (3.12) averaged across all replications.

Three major results emerge. First, the LMM offers considerable precision gains when compared to both benchmarks. Second, a rising instantaneous signal correlation $\rho$ is associated with a declining RMSE of the LMM, which is due to the decreasing variance, and thus confirms the findings from Section 3 in a realistic setting. Finally, the adaptive LMM closely tracks its oracle counterpart.

In summary, the simulation results show that the estimator has promising properties even in settings which are more general than those assumed in $\left(\mathcal{E}_{1}\right)$, allowing, for instance, for random observation times, stochastic intraday volatility as well as leverage effects. Even if the latter effects are not yet covered by our theory, the proposed estimator seems to be quite robust to deviations from the idealised setting. 


\section{APPENDIX A: FROM DISCRETE TO CONTINUOUS EXPERIMENTS}

Proof of Theorem 2.4. To establish Le Cam equivalence, we give a constructive proof to transfer observations in $\mathcal{E}_{0}$ to the continuous-time model $\mathcal{E}_{1}$ and the other way round. We bound the Le Cam distance by estimates for the squared Hellinger distance between Gaussian measures and refer to Section A.1 in [19] for information on Hellinger distances between Gaussian measures and bounds with the Hilbert-Schmidt norm. The crucial difference here is that linear interpolation is carried out for non-synchronous irregular observation schemes. Consider the linear B-splines or hat functions

$$
b_{i, n}(t)=\mathbb{1}_{\left[\frac{i-1}{n}, \frac{i+1}{n}\right]}(t) \min \left(1+n\left(t-\frac{i}{n}\right), 1-n\left(t-\frac{i}{n}\right)\right) .
$$

Define $b_{i}^{l}(t):=b_{i, n_{l}}\left(F_{l}(t)\right), 1 \leq i \leq n_{l}, 1 \leq l \leq d$, which are warped spline functions satisfying $b_{i_{1}}^{l}\left(t_{i_{2}}^{(l)}\right)=\delta_{i_{1}, i_{2}}$. A centered Gaussian process $\hat{Y}$ is derived from linearly interpolating each component of $Y$ :

$$
\hat{Y}_{t}^{(l)}=\sum_{i=1}^{n_{l}} Y_{i}^{(l)} b_{i}^{l}(t)=\sum_{i=1}^{n_{l}} X_{t_{i}^{(l)}}^{(l)} b_{i}^{l}(t)+\sum_{i=1}^{n_{l}} \varepsilon_{i}^{(l)} b_{i}^{l}(t) .
$$

The covariance matrix function $\mathbb{E}\left[\hat{Y}_{t} \hat{Y}_{s}^{\top}\right]$ of the interpolated process $\hat{Y}$ is determined by

$$
\begin{aligned}
\mathbb{E}\left[\hat{Y}_{t}^{(l)} \hat{Y}_{s}^{(r)}\right] & =\sum_{i=1}^{n_{l}} \sum_{\nu=1}^{n_{r}} a_{l r}\left(t_{i}^{(l)} \wedge t_{\nu}^{(r)}\right) b_{i}^{l}(t) b_{\nu}^{r}(s)+\delta_{l, r} \eta_{l}^{2} \sum_{i=1}^{n_{l}} b_{i}^{l}(t) b_{i}^{l}(s) \\
\text { with } A(t) & =\left(a_{l r}(t)\right)_{l, r=1, \ldots, d}=\int_{0}^{t} \Sigma(s) d s
\end{aligned}
$$

For any $g=\left(g^{(1)}, \ldots, g^{(d)}\right)^{\top} \in L^{2}\left([0,1], \mathbb{R}^{d}\right)$ we have

$$
\begin{aligned}
\mathbb{E}\left[\langle\mathbf{g}, \hat{Y}\rangle^{2}\right] & =\mathbb{E}\left[\left(\sum_{\nu=1}^{d}\left\langle g^{(v)}, \hat{Y}^{(v)}\right\rangle\right)^{2}\right] \\
& =\sum_{l, r=1}^{d} \sum_{i=1}^{n_{l}} \sum_{\nu=1}^{n_{r}} a_{l r}\left(t_{i}^{(l)} \wedge t_{\nu}^{(r)}\right)\left\langle g^{(l)}, b_{i}^{l}\right\rangle\left\langle g^{(r)}, b_{\nu}^{r}\right\rangle+\sum_{l=1}^{d} \sum_{i=1}^{n_{l}}\left\langle g^{(l)}, b_{i}^{l}\right\rangle^{2} \eta_{l}^{2} .
\end{aligned}
$$

The sum of the addends induced by the observation noise in diagonal terms is bounded from above by

$$
\sum_{l=1}^{d} \frac{\eta_{l}^{2}}{n_{l}}\left\|g^{(l)} / \sqrt{F_{l}^{\prime}}\right\|_{L^{2}}^{2}=\sum_{l=1}^{d}\left\|g^{(l)} H_{n, l}\right\|_{L^{2}}^{2},
$$


since by virtue of $0 \leq \sum_{i} b_{i, n} \leq 1, \int b_{i, n}=1 / n$ and Jensen's inequality:

$$
\begin{aligned}
\sum_{i=1}^{n_{l}}\left\langle g^{(l)}, b_{i}^{l}\right\rangle^{2} & \leq \frac{1}{n_{l}} \sum_{i=1}^{n_{l}} \int_{0}^{1}\left(\left(g^{(l)} \circ F_{l}^{-1}\right) \cdot\left(F_{l}^{-1}\right)^{\prime}\right)^{2} b_{i, n_{l}} \\
& \leq \frac{1}{n_{l}} \int_{0}^{1}\left(\left(g^{(l)} \circ F_{l}^{-1}\right) \cdot\left(F_{l}^{-1}\right)^{\prime}\right)^{2}=\frac{1}{n_{l}} \int_{0}^{1} \frac{\left(g^{(l)}\right)^{2}}{F_{l}^{\prime}} .
\end{aligned}
$$

On the other hand, we have

$$
\mathbb{E}\left[\left\langle g, \operatorname{diag}\left(H_{n, l}\right)_{l} d W\right\rangle\right]=\sum_{l=1}^{d}\left\|g^{(l)} H_{n, l}\right\|_{L^{2}}^{2}
$$

for a $d$-dimensional standard Brownian motion $W$. Consequently, a process $\bar{Y}$ with continuous-time white noise and the same signal part as $\hat{Y}$ can be obtained by adding uninformative noise. Introduce the process

$$
d \bar{Y}=\left(\sum_{i=1}^{n_{l}} X_{t_{i}^{(l)}} b_{i}^{l}(t)\right)_{1 \leq l \leq d} d t+\operatorname{diag}\left(H_{n, l}(t)\right)_{1 \leq l \leq d} d W_{t},
$$

and its associated covariance operator $\bar{C}: L^{2} \rightarrow L^{2}$, given by

$$
\bar{C} g(t)=\left(\sum_{r=1}^{d} \sum_{i=1}^{n_{l}} \sum_{\nu=1}^{n_{r}} a_{l r}\left(t_{i}^{(l)} \wedge t_{\nu}^{(r)}\right)\left\langle g^{(r)}, b_{\nu}^{r}\right\rangle\right)_{1 \leq l \leq d}+\left(H_{n, l}(t)^{2} g^{(l)}(t)\right)_{1 \leq l \leq d} .
$$

In fact, it is possible to transfer observations from our original experiment $\mathcal{E}_{0}$ to observations of (A.2) by adding $\mathbf{N}(0, \bar{C}-\hat{C})$-noise, where $\hat{C}: L^{2} \rightarrow L^{2}$ is the covariance operator of $\hat{Y}$. Now, consider the covariance operator

$$
C g(t)=\int_{0}^{1}\left(\int_{0}^{t \wedge u} A(s) d s\right) g(u) d u+\left(\frac{\eta_{l}^{2}}{n_{l} F_{l}^{\prime}(t)} g^{(l)}(t)\right)_{1 \leq l \leq d},
$$

associated with the continuous-time experiment $\mathcal{E}_{1}$.

We can bound $C^{-1 / 2}$ on $L^{2}\left([0,1], \mathbb{R}^{d}\right)$ from below (by partial ordering of operators) by a simple matrix multiplication operator:

$$
C^{-1 / 2} \leq \mathcal{M}_{\operatorname{diag}\left(H_{n, l}(t)\right)_{l}} .
$$

Denote the Hilbert-Schmidt norm by $\|\cdot\|_{\text {HS }}$. The asymptotic equivalence of observing $\bar{Y}$ and $Y$ in $\mathcal{E}_{1}$ is ensured by the Hellinger distance bound

$$
\mathrm{H}^{2}(\mathcal{L}(\bar{Y}), \mathcal{L}(Y)) \leq 2\left\|C^{-1 / 2}(\bar{C}-C) C^{-1 / 2}\right\|_{\mathrm{HS}}^{2}
$$




$$
\begin{aligned}
\leq & 2 \int_{0}^{1} \int_{0}^{1}\left(\sum_{l=1}^{d} \sum_{r=1}^{d} H_{n, l}(t)^{-2} H_{n, r}(t)^{-2}\right. \\
& \left.\left(\sum_{i=1}^{n_{l}} \sum_{\nu=1}^{n_{r}} a_{l r}\left(t_{i}^{(l)} \wedge t_{\nu}^{(r)}\right) b_{i}^{l}(t) b_{\nu}^{r}(s)-a_{l r}(t \wedge s)\right)^{2}\right) d t d s \\
= & 2 \int_{0}^{1} \int_{0}^{1}\left(\sum_{l=1}^{d} \sum_{r=1}^{d} \frac{n_{l} n_{r}}{\eta_{l}^{2} \eta_{r}^{2}}\right. \\
& \left(\sum_{i=1}^{n_{l}} \sum_{\nu=1}^{n_{r}} a_{l r}\left(t_{i}^{(l)} \wedge t_{\nu}^{(r)}\right) b_{i, n_{l}}(u) b_{\nu, n_{r}}(z)-a_{l r}\left(F_{l}^{-1}(u) \wedge F_{r}^{-1}(z)\right)^{2}\right) d u d z \\
= & \mathcal{O}\left(R^{4} \sum_{l=1}^{d} \sum_{r=1}^{d} \eta_{l}^{-2} \eta_{r}^{-2} n_{l} n_{r} n_{\min }^{-2-2 \beta}\right) .
\end{aligned}
$$

The estimate for the $L^{2}$-distance between the function $(t, s) \mapsto A\left(F_{l}^{-1}(t) \wedge\right.$ $\left.F_{r}^{-1}(s)\right),(l, r) \in\{1, \ldots, d\}^{2}$, and its coordinate-wise linear interpolation by $\mathcal{O}\left(n_{\min }^{-1-\beta} \vee n_{\min }^{-3 / 2}\right)$ relies on a standard approximation result on a rectangular grid of maximal width $\left(n_{\min }\right)^{-1}$ based on the fact that this function lies in the Sobolev class $H^{1+\beta}\left([0,1]^{2}\right)$ with corresponding norm bounded by $2 R^{4}$. This follows immediately by the product rule from $A^{\prime}=\Sigma \in H^{\beta}$ and $\left(F_{l}^{-1}\right)^{\prime} \in C^{\beta}$, together with an $L^{2}$-error bound at the skewed diagonal $\left\{(t, s): F_{l}(t)=F_{r}(s)\right\}$.

Next, we explicitly show that $\mathcal{E}_{1}$ is at least as informative as $\mathcal{E}_{0}$. To this end we discretise in each component on the intervals $I_{i, l}=\left[\frac{i}{n_{l}}-\frac{1}{2 n_{l}}, \frac{i}{n_{l}}+\right.$ $\left.\frac{1}{2 n_{l}}\right] \cap[0,1]$ for $i=0, \ldots, n_{l}$. Define

$$
\begin{aligned}
\left(Y_{i}^{\prime}\right)^{(l)}=\frac{1}{\left|I_{i, l}\right|} \int_{F_{l}^{-1}\left(I_{i, l}\right)} F_{l}^{\prime}(t) d Y_{t}^{(l)} & =\frac{1}{\left|I_{i, l}\right|} \int_{F_{l}^{-1}\left(I_{i, l}\right)} X_{t}^{(l)} F_{l}^{\prime}(t) d t+\varepsilon_{i}^{(l)} \\
& =\frac{1}{\left|I_{i, l}\right|} \int_{I_{i, l}} X_{F^{-1}(u)}^{(l)} d u+\varepsilon_{i}^{(l)}
\end{aligned}
$$

for $0 \leq i \leq n_{l}$ with i. i. d. random variables:

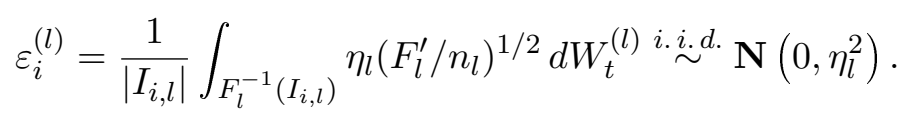

The covariances are calculated as

$$
\mathbb{E}\left[\left(Y_{i}^{\prime}\right)^{(l)}\left(Y_{\nu}^{\prime}\right)^{(r)}\right]=\frac{1}{\left|I_{i, l}\right|\left|I_{\nu, r}\right|} \int_{I_{i, l}} \int_{I_{\nu, r}} a_{l r}\left(F_{l}^{-1}(u) \wedge F_{r}^{-1}\left(u^{\prime}\right)\right) d u d u^{\prime}+\delta_{l, r} \delta_{i, \nu} \eta_{l}^{2}
$$

We obtain for the squared Hellinger distance between the laws of observation

$$
\mathrm{H}^{2}\left(\mathcal{L}\left(\left(Y_{i}^{(l)}\right)_{l=1, \ldots, d ; i=0, \ldots, n_{l}}\right), \mathcal{L}\left(\left(\left(Y_{i}^{\prime}\right)^{(l)}\right)_{l=1, \ldots, d ; i=0, \ldots, n_{l}}\right)\right)
$$




$$
\begin{aligned}
\leq \sum_{l, r=1}^{d} \eta_{l}^{-2} \eta_{r}^{-2} \sum_{i=0}^{n_{l}} \sum_{\nu=0}^{n_{r}}\left(\frac{1}{\left|I_{i, l}\right|\left|I_{\nu, r}\right|} \int_{I_{i, l}} \int_{I_{\nu, r}} a_{l r}\left(F_{l}^{-1}(u) \wedge F_{r}^{-1}\left(u^{\prime}\right)\right)\right. \\
\left.-a_{l r}\left(F_{l}^{-1}\left(i / n_{l} \wedge \nu / n_{r}\right)\right) d u d u^{\prime}\right)^{2} .
\end{aligned}
$$

Write $A_{l r}^{F}\left(u, u^{\prime}\right)=a_{l r}\left(F_{l}^{-1}(u) \wedge F_{r}^{-1}\left(u^{\prime}\right)\right)$ and note $A_{l r}^{F} \in H^{1+\beta}\left([0,1]^{2}\right)$ due to $A^{\prime}=\Sigma \in H^{\beta}$ and $F_{l}^{-1}, F_{r}^{-1} \in C^{\beta}$. For $(i, \nu) \notin \mathcal{C}:=\left\{(0,0),\left(0, n_{r}\right),\left(n_{l}, 0\right)\right.$, $\left.\left(n_{l}, n_{r}\right)\right\}$ the rectangle $I_{i, l} \times I_{\nu, r}$ is symmetric around $\left(i / n_{l}, \nu / n_{r}\right)$ such that the integral in the preceding display equals ( $\nabla$ denotes the gradient)

$$
\begin{aligned}
& \int_{I_{i, l} \times I_{\nu, r}} \int_{0}^{1}\left(\left\langle\nabla A_{l r}^{F}\left(\frac{i}{n_{l}}+\vartheta\left(u-\frac{i}{n_{l}}\right), \frac{\nu}{n_{r}}+\vartheta\left(u^{\prime}-\frac{\nu}{n_{r}}\right)\right),\left(u-\frac{i}{n_{l}}, u^{\prime}-\frac{\nu}{n_{r}}\right)\right\rangle\right. \\
& \left.-\left\langle\nabla A_{l r}^{F}\left(\frac{i}{n_{l}}, \frac{\nu}{n_{r}}\right),\left(u-\frac{i}{n_{l}}, u^{\prime}-\frac{\nu}{n_{r}}\right)\right\rangle\right) d \vartheta d u d u^{\prime} .
\end{aligned}
$$

Using Jensen's inequality we thus obtain further the bound for the squared Hellinger distance:

$$
\begin{gathered}
\sum_{l, r=1}^{d} \eta_{l}^{-2} \eta_{r}^{-2} \sum_{i=0}^{n_{l}} \sum_{\nu=0}^{n_{r}} \frac{\left(n_{l} \vee n_{r}\right)^{-2}}{\left|I_{i, l}\right|\left|I_{\nu, r}\right|} \int_{I_{i, l} \times I_{\nu, r}} \int_{0}^{1} \| \nabla A_{l r}^{F}\left(i / n_{l}+\vartheta\left(u-i / n_{l}\right),\right. \\
\left.\nu / n_{r}+\vartheta\left(u^{\prime}-\nu / n_{r}\right)\right)-\nabla A_{l r}^{F}\left(i / n_{l}, \nu / n_{r}\right) \mathbb{1}((i, \nu) \notin \mathcal{C}) \|^{2} d \vartheta d u d u^{\prime} \\
=\sum_{l, r=1}^{d} \eta_{l}^{-2} \eta_{r}^{-2} \frac{n_{l} n_{r}}{\left(n_{l} \vee n_{r}\right)^{2}} \mathcal{O}\left(R^{4}\left(n_{l} \wedge n_{r}\right)^{-2 \beta}\right)=\mathcal{O}\left(R^{4}\left(\sum_{l=1}^{d} n_{l} / \eta_{l}^{2}\right)^{2} n_{\text {min }}^{-2 \beta}\right)
\end{gathered}
$$

where the order estimate is due to $\left\|\nabla A_{l r}^{F}\right\|_{H^{\beta}} \leq R^{2}$ and a standard $L^{2}$ approximation result for Sobolev spaces, observing that for the four corner rectangles in $\mathcal{C}$ the boundedness of the respective integrals only adds the total order $4 n_{\text {min }}^{-2}<n_{l} n_{r} n_{\text {min }}^{-2-2 \beta}$.

\section{APPENDIX B: ASYMPTOTICS IN THE BLOCK-WISE CONSTANT EXPERIMENT}

Proof of TheOrem 3.2. As we have seen, the estimator is unbiased in $\mathcal{E}_{2}$. For the covariance structure we use the independence between blocks and frequencies and the commutativity with $\mathcal{Z}$ to infer

$$
\begin{aligned}
\operatorname{COV}_{\mathcal{E}_{2}}\left(\mathbf{I}_{n}^{1 / 2} \operatorname{LMM}_{\text {or }}^{(n)}\right) & =\mathbf{I}_{n}^{1 / 2} \sum_{k=0}^{h^{-1}-1} h^{2} \sum_{j=1}^{\infty} W_{j k} \operatorname{COV}_{\mathcal{E}_{2}}\left(\operatorname{vec}\left(S_{j k} S_{j k}^{\top}\right)\right) W_{j k}^{\top} \mathbf{I}_{n}^{1 / 2} \\
& =\mathbf{I}_{n}^{1 / 2} \sum_{k=0}^{h^{-1}-1} h^{2} I_{k}^{-1} \mathbf{I}_{n}^{1 / 2} \mathcal{Z}=\mathcal{Z}
\end{aligned}
$$


Since the local Fisher-type informations are strictly positive definite and thus invertible by Assumption 2.2(iii), the multivariate CLT (3.10) for the oracle estimator follows by applying a standard CLT for triangular schemes as Theorem 4.12 from [14]. The Lindeberg condition is implied by the stronger Lyapunov condition which is easily verified here by bounding moments of order 4 .

In Appendix $\mathrm{C}$ below we prove that in experiment $\mathcal{E}_{1}$ the estimator $\mathrm{LMM}_{o r}^{(n)}$ has an additional bias of order $\mathcal{O}\left(n_{\text {min }}^{-\alpha / 2}\right)+\mathcal{O}_{P}(h)$ and a difference in the covariance of order $\mathcal{O}\left(h n_{\min }^{-\alpha / 2}\right)+\mathcal{O}_{P}\left(h^{2}\right)$ under our Assumption $2.2(i i-\alpha),(i i i-\underline{\Sigma})$, which by Slutsky's lemma yields an asymptotically negligible term compared to the best attainable rate (in any entry) $n_{\max }^{-1 / 4}$, cf. Theorem 4.3.

Proof of Corollary 3.3. An important property of our oracle estimator is its equivariance with respect to invertible linear transformations $A_{k}$ on each block $k$ in the sense that for observed statistics $\tilde{S}_{j k}:=A_{k} S_{j k} \sim$ $\mathbf{N}\left(0, \tilde{C}_{j k}\right)$ under $\mathcal{E}_{2}$ we obtain $\left(A^{-\top}:=\left(A^{\top}\right)^{-1}\right.$ for short $)$

$C_{j k}=A_{k}^{-1} \tilde{C}_{j k} A_{k}^{-\top}, I_{j k}=\left(A_{k} \otimes A_{k}\right)^{\top} \tilde{I}_{j k}\left(A_{k} \otimes A_{k}\right), I_{k}=\left(A_{k} \otimes A_{k}\right)^{\top} \tilde{I}_{k}\left(A_{k} \otimes A_{k}\right)$

and hence

$$
\begin{aligned}
\operatorname{LMM}_{o r}^{(n)} & =\sum_{k=0}^{h^{-1}-1} h\left(A_{k} \otimes A_{k}\right)^{-1} \tilde{I}_{k}^{-1} \sum_{j \geq 0} \tilde{I}_{j k}\left(A_{k} \otimes A_{k}\right) \operatorname{vec}\left(S_{j k} S_{j k}^{\top}\right) \\
& =\sum_{k=0}^{h^{-1}-1}\left(A_{k} \otimes A_{k}\right)^{-1}\left(h \tilde{I}_{k}^{-1} \sum_{j \geq 0} \tilde{I}_{j k} \operatorname{vec}\left(\tilde{S}_{j k} \tilde{S}_{j k}^{\top}\right)\right) .
\end{aligned}
$$

For the covariance we use commutativity with $\mathcal{Z}$ and obtain likewise

$$
\operatorname{COV}_{\mathcal{E}_{2}}\left(\mathrm{LMM}_{\text {or }}^{(n)}\right)=\sum_{k=0}^{h^{-1}-1} h^{2}\left(A_{k} \otimes A_{k}\right)^{-1} \tilde{I}_{k}^{-1}\left(A_{k} \otimes A_{k}\right)^{-\top} \mathcal{Z}
$$

We use this property to diagonalise the problem on each block. In terms of the noise level matrix $\mathcal{H}_{k}:=\operatorname{diag}\left(H_{l, n}^{k}\right)_{l=1, \ldots, d}$, let $O_{k}$ be an orthogonal matrix such that

$$
\Lambda^{k h}=O_{k} \mathcal{H}_{k}^{-1} \Sigma^{k h} \mathcal{H}_{k}^{-1} O_{k}^{\top}
$$

is diagonal. Note that $\Lambda^{k h}$ grows with $n$, but we drop the dependence on $n$ in the notation for all matrices $\Lambda^{k h}, O_{k}$ and $\mathcal{H}_{k}$. Use $A_{k}=O_{k} \mathcal{H}_{k}^{-1}$ to obtain the spectral statistics (3.2) transformed:

$$
\tilde{S}_{j k}=O_{k} \mathcal{H}_{k}^{-1} S_{j k} \sim \mathbf{N}\left(\mathbf{0}, \tilde{C}_{j k}\right) \text { independent for all }(j, k)
$$


which yields a simple-structured diagonal covariance matrix:

$$
\tilde{C}_{j k}=O_{k} \mathcal{H}_{k}^{-1} C_{j k} \mathcal{H}_{k}^{-1} O_{k}^{\top}=\Lambda^{k h}+\frac{\pi^{2} j^{2}}{h^{2}} E_{d}
$$

A key point is that the covariance structure (3.7) in $\mathbb{R}^{d^{2} \times d^{2}}$ is for independent components $\tilde{S}_{j k}$ also diagonal, up to symmetry in the volatility matrix entries. Summing $\tilde{I}_{j k}$ over $j$ is explicitly solvable and gives for $p, q=1, \ldots, d$

$$
\begin{aligned}
& \left(h \tilde{I}_{k}^{-1}\right)_{p, q}=\left(h^{-1} \sum_{j=1}^{\infty}\left(\tilde{C}_{j k}^{-1} \otimes \tilde{C}_{j k}^{-1}\right)_{p, q}\right)^{-1} \\
& =\left(h^{-1} \sum_{j=1}^{\infty}\left(\Lambda_{p p}^{k h}+\pi^{2} j^{2} h^{-2}\right)^{-1}\left(\Lambda_{q q}^{k h}+\pi^{2} j^{2} h^{-2}\right)^{-1}\right)^{-1} \\
& =\left(\frac{\sqrt{\Lambda_{q q}^{k h}} \operatorname{coth}\left(h \sqrt{\Lambda_{p p}^{k h}}\right)-\sqrt{\Lambda_{p p}^{k h}} \operatorname{coth}\left(h \sqrt{\Lambda_{q q}^{k h}}\right)}{2 \sqrt{\Lambda_{p p}^{k h} \Lambda_{q q}^{k h}}\left(\Lambda_{q q}^{k h}-\Lambda_{p p}^{k h}\right)}-\frac{1}{2 h \Lambda_{p p}^{k h} \Lambda_{q q}^{k h}}\right)^{-1} \\
& =2\left(\Lambda_{p p}^{k h} \sqrt{\Lambda_{q q}^{k h}}+\Lambda_{q q}^{k h} \sqrt{\Lambda_{p p}^{k h}}\right)\left(1+\mathcal{O}\left(e^{-2 h \sqrt{\Lambda_{p p}^{k h} \wedge \Lambda_{q q}^{k h}}}+h^{-1}\left(\Lambda_{p p}^{k h} \wedge \Lambda_{q q}^{k h}\right)^{-1 / 2}\right)\right),
\end{aligned}
$$

using $\Lambda^{k h} \geq\left(\min _{l, t} n_{l} F_{l}^{\prime}(t) \eta_{l}^{-2}\right) \underline{\Sigma} \gtrsim n_{\min } E_{d}, h^{2} n_{\text {min }} \rightarrow \infty$ and $\operatorname{coth}(x)=$ $1+\mathcal{O}\left(e^{-2 x}\right)$ for $x \rightarrow \infty$. We thus obtain uniformly over $k$

$$
h \tilde{I}_{k}^{-1}=(2+\mathcal{O}(1))\left(\Lambda^{k h} \otimes \sqrt{\Lambda^{k h}}+\sqrt{\Lambda^{k h}} \otimes \Lambda^{k h}\right) .
$$

By formula (B.2) we infer in terms of $\left(\Sigma_{\mathcal{H}}^{k h}\right)^{1 / 2}:=\mathcal{H}_{k}\left(\mathcal{H}_{k}^{-1} \Sigma^{k h} \mathcal{H}_{k}^{-1}\right)^{1 / 2} \mathcal{H}_{k}$

$$
\operatorname{COV}_{\mathcal{E}_{2}}\left(\mathrm{LMM}_{\text {or }}^{(n)}\right)=(2+\mathcal{O}(1)) \sum_{k=0}^{h^{-1}-1} h\left(\Sigma^{k h} \otimes\left(\Sigma_{\mathcal{H}}^{k h}\right)^{1 / 2}+\left(\Sigma_{\mathcal{H}}^{k h}\right)^{1 / 2} \otimes \Sigma^{k h}\right) \mathcal{Z}
$$

The final step consists in combining $n_{\text {min }}^{1 / 2} H_{n, l}(t) \rightarrow H_{l}(t)$ uniformly in $t$ together with a Riemann sum approximation to conclude

$$
\begin{aligned}
& \lim _{n_{\text {min }} \rightarrow \infty} n_{\text {min }}^{1 / 2} \operatorname{COV}_{\mathcal{E}_{2}}\left(\mathrm{LMM}_{\text {or }}^{(n)}\right) \\
& \quad=2\left(\int_{0}^{1}\left(\Sigma \otimes\left(\mathcal{H}\left(\mathcal{H}^{-1} \Sigma \mathcal{H}^{-1}\right)^{1 / 2} \mathcal{H}\right)+\left(\mathcal{H}\left(\mathcal{H}^{-1} \Sigma \mathcal{H}^{-1}\right)^{1 / 2} \mathcal{H}\right) \otimes \Sigma\right)(t) d t\right) \mathcal{Z}
\end{aligned}
$$

\section{APPENDIX C: PROOFS FOR CONTINUOUS MODELS}

C.1. Weight matrix estimates. We shall often need general norm bounds on the weight matrices $W_{j k}$. 
LEMMA C.1. The oracle weight matrices satisfy uniformly over $(j, k)$ and the matrices $\Sigma^{k h}$ with $\left\|\Sigma^{k h}\right\|_{\infty}+\left\|\left(\Sigma^{k h}\right)^{-1}\right\|_{\infty} \lesssim 1$

$$
\left\|W_{j k}\right\| \lesssim h_{0}^{-1}\left(1+j^{4} / h_{0}^{4}\right)^{-1} \text {. }
$$

Proof. From the proof of Corollary 3.3 we infer

$$
\begin{aligned}
& W_{j k}=\left(H_{k} O_{k}^{\top} \otimes H_{k} O_{k}^{\top}\right) \tilde{W}_{j k}\left(O_{k} H_{k}^{-1} \otimes\left(O_{k} H_{k}^{-1}\right)\right. \text { with } \\
& \tilde{W}_{j k}=(2+\mathcal{O}(1)) h^{-1}\left(\left(\Lambda^{k h} \tilde{C}_{j k}^{-1}\right) \otimes\left(\sqrt{\Lambda^{k h}} \tilde{C}_{j k}^{-1}\right)+\left(\sqrt{\Lambda^{k h}} \tilde{C}_{j k}^{-1}\right) \otimes\left(\Lambda^{k h} \tilde{C}_{j k}^{-1}\right)\right) .
\end{aligned}
$$

We evaluate one factor in $W_{j k}$ using

$\left\|H_{k} O_{k}^{\top} \Lambda^{k h} \tilde{C}_{j k}^{-1} O_{k} H_{k}^{-1}\right\|=\left\|\Sigma^{k h}\left(\Sigma^{k h}+\pi^{2} j^{2} h^{-2} H_{k}^{2}\right)^{-1}\right\| \lesssim\left(1+j^{2} h^{-2} n_{\text {min }}^{-2}\right)^{-1}$.

By $\|A \otimes B\| \leq\|A\|\|B\|$ and $\sqrt{\Lambda^{k h}} \tilde{C}_{j k}^{-1}=\left(\Lambda^{k h} \tilde{C}_{j k}^{-1}\right)\left(\Lambda^{k h}\right)^{-1 / 2}$ (the matrices are diagonal), we infer

$$
\left\|W_{j k}\right\| \lesssim h^{-1}\left(1+j^{2} h_{0}^{-2}\right)^{-2}\left\|H_{k} O_{k}^{\top}\left(\Lambda^{k h}\right)^{-1 / 2} O_{k} H_{k}^{-1}\right\| .
$$

To evaluate the last norm, despite matrix multiplication is non-commutative, we note

$$
\left(O_{k}^{\top}\left(\Lambda^{k h}\right)^{-\frac{1}{2}} O_{k} H_{k}^{-1}\right)^{\top} O_{k}^{\top}\left(\Lambda^{k h}\right)^{-\frac{1}{2}} O_{k} H_{k}^{-1}=H_{k}^{-1} O_{k}^{\top}\left(\Lambda^{k h}\right)^{-1} O_{k} H_{k}^{-1}=\left(\Sigma^{k h}\right)^{-1},
$$

whence by polar decomposition $\left|O_{k}^{\top}\left(\Lambda^{k h}\right)^{-1 / 2} O_{k} H_{k}^{-1}\right|=\left(\Sigma^{k h}\right)^{-1 / 2}$ implies

$$
\left\|O_{k}^{\top}\left(\Lambda^{k h}\right)^{-\frac{1}{2}} O_{k} H_{k}^{-1}\right\|=\left\|\left(\Sigma^{k h}\right)^{-\frac{1}{2}}\right\| \lesssim 1 .
$$

Together with $\left\|H_{k}\right\| \lesssim n_{\text {min }}^{-1 / 2}$ this yields

$$
\left\|W_{j k}\right\| \lesssim h^{-1}\left(1+j^{2} h_{0}^{-2}\right)^{-2} n_{\min }^{-1 / 2}
$$

which gives the result.

Moreover, for the adaptive estimator we have to control the dependence of the weight matrices $W_{j k}=W_{j}\left(\Sigma^{k h}\right)$ on $\Sigma^{k h}$. We use the notion of matrix differentiation as introduced in [9]: define the derivative $d A / d B$ of a matrixvalued function $A(B) \in \mathbb{R}^{o \times p}$ with respect to $B \in \mathbb{R}^{q \times r}$ as the $\mathbb{R}^{o p \times q r}$ matrix with row vectors $\left(d / d B_{a b}\right) \operatorname{vec}(A), 1 \leq a \leq q, 1 \leq b \leq r$.

Lemma C.2. For the derivatives of the oracle weight matrices $W_{j}\left(\Sigma^{k h}\right)$, assuming $\left\|\Sigma^{k h}\right\|_{\infty}+\left\|\left(\Sigma^{k h}\right)^{-1}\right\|_{\infty} \lesssim 1$, we have uniformly over $(j, k)$ :

$$
\left\|\frac{d}{d \Sigma^{k h}} W_{j}\left(\Sigma^{k h}\right)\right\| \lesssim h_{0}^{-1}\left(1+j^{4} h_{0}^{-4}\right)^{-1}
$$


Proof. Since the notion of matrix derivatives relies on vectorisation, the identities $\operatorname{vec}\left(I_{k}^{-1} I_{j k}\right)=\left(E_{d^{2}} \otimes I_{k}^{-1}\right) \operatorname{vec}\left(I_{j k}\right)=\left(I_{j k}^{\top} \otimes E_{d^{2}}\right) \operatorname{vec}\left(I_{k}^{-1}\right)$ give rise to the matrix differentiation product rule

$$
\frac{d}{d \Sigma^{k h}} W_{j k}=\left(I_{j k} \otimes E_{d^{2}}\right) \frac{d I_{k}^{-1}}{d \Sigma^{k h}}+\left(E_{d^{2}} \otimes I_{k}^{-1}\right) \frac{d I_{j k}}{d \Sigma^{k h}}
$$

Applying the mixed product rule $(A \otimes B)(C \otimes D)=(A C \otimes B D)$ repeatedly, and the differentiation product rule and chain rule to $I_{j k}=C_{j k}^{-1} \otimes C_{j k}^{-1}$, we obtain

$$
\begin{aligned}
& \frac{d}{d C_{j k}}\left(C_{j k}^{-1} \otimes C_{j k}^{-1}\right)=-\left(\left(C_{j k}^{-1} \otimes C_{j k}^{-1}\right) \otimes\left(C_{j k}^{-1} \otimes C_{j k}^{-1}\right)\right)\left(\left(\left(C_{j k} \otimes E_{d} \otimes E_{d^{2}}\right)\right.\right. \\
& \left.\left.+\left(E_{d^{2}} \otimes E_{d} \otimes C_{j k}\right)\right)\left(E_{d} \otimes C_{d, d} \otimes E_{d}\right)\left(\left(\operatorname{vec}\left(E_{d}\right) \otimes E_{d^{2}}\right)+\left(E_{d^{2}} \otimes \operatorname{vec}\left(E_{d}\right)\right)\right)\right),
\end{aligned}
$$

with the so-called commutation matrix $C_{d, d}=\mathcal{Z}-E_{d^{2}}$. By orthogonality of the last factors in both addends, $\|A \otimes B\|=\|A\|\|B\|$, and the mixed product rule, we infer for the norm of the second addend in (C.2)

$$
\begin{aligned}
\left\|\left(E_{d^{2}} \otimes I_{k}^{-1}\right) \frac{d I_{j k}}{d \Sigma^{k h}}\right\| & \leq 2\left\|\left(E_{d} \otimes C_{j k}^{-1}\right) \otimes\left(I_{k}^{-1}\left(C_{j k}^{-1} \otimes C_{j k}^{-1}\right)\right)\right\| \\
& =2\left\|W_{j k}\right\|\left\|C_{j k}^{-1}\right\| \lesssim\left\|W_{j k}\right\| .
\end{aligned}
$$

By virtue of

$$
\left(I_{k}^{-1} \otimes E_{d^{2}}\right) \frac{d I_{k}}{d \Sigma^{k h}}=-\left(E_{d^{2}} \otimes I_{k}\right) \frac{d I_{k}^{-1}}{d \Sigma^{k h}},
$$

it follows with the mixed product rule that

$$
d I_{k}^{-1} / d \Sigma^{k h}=-\left(I_{k}^{-1} \otimes I_{k}^{-1}\right)\left(d I_{k} / d \Sigma^{k h}\right) .
$$

This yields for the norm of the first addend in (C.2)

$$
\begin{aligned}
\left\|\left(I_{j k} \otimes E_{d^{2}}\right) \frac{d I_{k}^{-1}}{d \Sigma^{k h}}\right\|=\left\|\left(W_{j k}^{\top} \otimes I_{k}^{-1}\right) \frac{d I_{k}}{d \Sigma^{k h}}\right\| & \lesssim\left\|W_{j k}\right\|\left\|\left(E_{d^{2}} \otimes I_{k}^{-1}\right) \sum_{j^{\prime}} \frac{d I_{j^{\prime} k}}{d \Sigma^{k h}}\right\| \\
& \lesssim\left\|W_{j k}\right\|\left(\sum_{j^{\prime}}\left\|W_{j^{\prime} k}\right\|\right) \lesssim\left\|W_{j k}\right\|
\end{aligned}
$$

since we can differentiate inside the sum by the absolute convergence of $\sum_{j^{\prime}}\left\|W_{j^{\prime} k}\right\|$. This proves our claim by Lemma C.1. 
C.2. Bias bound. Using the formula $1-2 \sin ^{2}(x)=\cos (2 x)$ and Itô isometry, the $(d \times d)$-matrix of (negative) biases (in the signal) of the addends in (3.8) as an estimator of $\Sigma^{k h}$ in experiment $\mathcal{E}_{1}$ is given by

$$
B_{j, k}:=2 h^{-1} \int_{k h}^{(k+1) h} \Sigma(t) \cos \left(2 j \pi h^{-1}(t-k h)\right) d t,
$$

which has the structure of a $j$-th Fourier cosine coefficient. We introduce the corresponding weighting function in the time domain:

$$
G_{k}(u)=2 \sum_{j=1}^{\infty} W_{j k} \cos (2 j \pi u) \in \mathbb{R}^{d^{2} \times d^{2}}, \quad u \in[0,1] .
$$

Parseval's identity then shows for the $d^{2}$-dimensional block-wise bias vector of (3.8):

$$
\sum_{j=1}^{\infty} W_{j k} \operatorname{vec}\left(B_{j, k}\right)=\int_{k h}^{(k+1) h} h^{-1} G_{k}\left(h^{-1}(t-k h)\right) \operatorname{vec}(\Sigma(t)) d t .
$$

The vector of total biases of (3.8) is then the linear functional of $\Sigma$ :

$$
\sum_{k=0}^{h^{-1}-1} h \sum_{j=1}^{\infty} W_{j k} \operatorname{vec}\left(B_{j k}\right)=\int_{0}^{1} G^{h}(t) \operatorname{vec}(\Sigma(t)) d t
$$

where for $t \in[k h,(k+1) h))$

$$
G^{h}(t)=G_{k}\left(h^{-1}(t-k h)\right)=2 \sum_{j=1}^{\infty} W_{j k} \cos \left(2 \pi j h^{-1} t\right) .
$$

For $\Sigma$ in the Besov space $B_{1, \infty}^{\alpha}([0,1]), 0<\alpha \leq 1$, the $L^{1}$-modulus of continuity satisfies $\omega_{L^{1}([0,1])}(\Sigma, \delta) \leq\|\Sigma\|_{B_{1, \infty}^{\alpha}} \delta^{\alpha}$, see e.g. [7, Section 3.2]. We have for $\delta \in(0,1)$ and $s \in[0,1-\delta]$

$$
\begin{aligned}
& \left|\int_{0}^{\delta} \operatorname{vec}(\Sigma(t+s)) \cos \left(\frac{2 \pi t}{\delta}\right) d t\right|=\frac{1}{\delta} \mid \int_{0}^{\delta} \int_{0}^{\delta} \operatorname{vec}(\Sigma(t+s)-\Sigma(u+s)) d u \\
& \cos \left(\frac{2 \pi t}{\delta}\right) d t\left|\leq \sup _{0 \leq v \leq \delta} \int_{0}^{\delta}\right| \operatorname{vec}(\Sigma(t+s)-\Sigma(t+v+s)) \mid d t \leq \omega_{L^{1}([s, s+\delta])}(\Sigma, \delta) .
\end{aligned}
$$

This shows for the total bias in estimation of the volatility in $X$ by the bound on $\left\|W_{j k}\right\|$ in Lemma C.1

$$
\begin{aligned}
\left|\int_{0}^{1} G^{h}(t) \operatorname{vec}(\Sigma(t)) d t\right| & \leq 2 \sum_{k=0}^{h^{-1}-1} \sum_{j=1}^{\infty}\left\|W_{j k}\right\| \omega_{L^{1}([k h,(k+1) h])}(\Sigma, h / j) \\
& \lesssim \sum_{j=1}^{\infty} h_{0}^{-1}\left(1+\left(h_{0} / j\right)^{4}\right)^{-1}(h / j)^{\alpha} \asymp\left(h / h_{0}\right)^{\alpha}=n_{m i n}^{-\alpha / 2}
\end{aligned}
$$


We thus have a bias of order $\mathcal{O}\left(n_{\min }^{-\alpha / 2}\right)$. Remark that it is quite surprising that this bias bound is independent of $h$, which is also at the heart of the quasi-maximum likelihood method [1].

If $\operatorname{vec}(\Sigma)$ is a (vector-valued) square-integrable martingale, then we use that martingale differences are uncorrelated and write for the total bias

$$
\int_{0}^{1} G^{h}(t) \operatorname{vec}(\Sigma(t)) d t=\int_{0}^{1} G^{h}(t) \operatorname{vec}\left(\Sigma(t)-\Sigma\left(\left\lfloor h^{-1} t\right\rfloor h\right)\right) d t
$$

using $\int G_{k}=0$. This expression is centred with covariance matrix

$$
\begin{gathered}
\sum_{k=0}^{h^{-1}-1} \int_{[k h,(k+1) h]^{2}} G_{k}\left(h^{-1}(t-k h)\right) \mathbb{E}\left[\operatorname{vec}(\Sigma(t)-\Sigma(k h)) \operatorname{vec}(\Sigma(s)-\Sigma(k h))^{\top}\right] \\
G_{k}\left(h^{-1}(s-k h)\right) d t d s .
\end{gathered}
$$

The expected value in the display is smaller than (in matrix ordering) $\mathbb{E}\left[\operatorname{vec}(\Sigma((k+1) h)-\Sigma(k h)) \operatorname{vec}(\Sigma((k+1) h)-\Sigma(k h))^{\top}\right]$. Because of $\left\|G_{k}\right\|_{\infty} \lesssim 1$ the covariance matrix (in any norm) is of order $\mathcal{O}\left(h^{2} \mathbb{E}\left[\|\Sigma(1)-\Sigma(0)\|^{2}\right]\right)=$ $\mathcal{O}\left(h^{2}\right)$.

If $\Sigma=\Sigma^{B}+\Sigma^{M}$ is the sum of a function $\Sigma^{B}$ in $B_{1, \infty}^{\alpha}([0,1])$ and a squareintegrable martingale $\Sigma^{M}$, then the preceding estimations apply for each summand and the total bias has maximal order $\mathcal{O}\left(n_{\text {min }}^{-\alpha / 2}\right)+\mathcal{O}_{P}(h)$.

C.3. Variance for general continuous-time model. The covariance for the estimator under model $\mathcal{E}_{1}$ can be calculated as under model $\mathcal{E}_{2}$, but we lose independence between different frequencies $j, j^{\prime}$ on the same block. For that we use the formula for Gaussian random vectors $A, B$

$\mathbb{C O V}\left(\operatorname{vec}\left(A A^{\top}\right), \operatorname{vec}\left(B B^{\top}\right)\right)=(\mathbb{C O V}(B, B) \otimes \mathbb{C O V}(A, B)+\mathbb{C O V}(A, A) \otimes$ $\mathbb{C O V}(A, B)+\mathbb{C O V}(A, B) \otimes \mathbb{C O V}(A, A)+\mathbb{C O V}(A, B) \otimes \mathbb{C O V}(B, B)) \mathcal{Z} / 4$,

obtained by polarisation. This implies

$$
\begin{aligned}
& \left\|\operatorname{COV}_{\mathcal{E}_{1}}\left(\mathrm{LMM}_{\text {or }}^{(n)}\right)-\operatorname{COV}_{\mathcal{E}_{2}}\left(\mathrm{LMM}_{\text {or }}^{(n)}\right)\right\| \\
& \lesssim \sum_{k=0}^{h^{-1}-1} h^{2} \sum_{j, j^{\prime}=1}^{\infty}\left\|W_{j^{\prime} k}\right\|\left\|W_{j k}\left(\operatorname{COV}_{\mathcal{E}_{1}}\left(S_{j k}, S_{j k}\right) \otimes \operatorname{COV}_{\mathcal{E}_{1}}\left(S_{j k}, S_{j^{\prime} k}\right)\right)\right\| .
\end{aligned}
$$

From Lemma C.1 and $\|A \otimes B\| \leq\|A\|\|B\|$ for matrices $A, B$ we infer that the series over $j, j^{\prime}$ is bounded in order by

$$
\begin{aligned}
& \sum_{j, j^{\prime}=1}^{\infty} h_{0}^{-2}\left(1+j^{\prime} / h_{0}\right)^{-4}\left(1+j / h_{0}\right)^{-2}\left(\left\|\int_{0}^{1}\left(\Sigma-\bar{\Sigma}_{h}\right)(t) \frac{\Phi_{j k}(t) \Phi_{j^{\prime} k}(t)}{\left\|\Phi_{j k}\right\|_{L^{2}}\left\|\Phi_{j^{\prime} k}\right\|_{L^{2}}} d t\right\|\right. \\
& \left.+\left\|\int_{0}^{1} \operatorname{diag}\left(H_{n, l}^{2}-\bar{H}^{2}{ }_{n, l, h}\right)(t) \varphi_{j k}(t) \varphi_{j^{\prime} k}(t) d t\right\|\right) .
\end{aligned}
$$


The identities $2 \cos (a) \cos (b)=\cos (a+b)+\cos (a-b), 2 \sin (a) \sin (b)=$ $\cos (a-b)-\cos (a+b)$ and the same bound as in Section C.2 imply for $\Sigma,\left(F_{1}^{\prime}\right)^{-1}, \ldots,\left(F_{d}^{\prime}\right)^{-1} \in B_{1, \infty}^{\alpha}([0,1])$ (note that even $\left(F_{l}^{\prime}\right)^{-1} \in C^{\alpha}([0,1])$ )

$$
\begin{gathered}
\left\|\int_{0}^{1}\left(\Sigma-\bar{\Sigma}_{h}\right)(t) \frac{\Phi_{j k}(t) \Phi_{j^{\prime} k}(t)}{\left\|\Phi_{j k}\right\|_{L^{2}}\left\|\Phi_{j^{\prime} k}\right\|_{L^{2}}} d t\right\| \lesssim \\
\quad h^{-1}\left(\frac{h}{j+j^{\prime}}+\frac{h\left(1-\delta_{j, j^{\prime}}\right)}{\left|j-j^{\prime}\right|}\right)^{\alpha} \\
\times\|\Sigma\|_{B_{1, \infty}^{\alpha}([k h,(k+1) h])}
\end{gathered}
$$

and similarly the bound

$$
h^{-1}\left(\frac{h}{j+j^{\prime}}+\frac{h\left(1-\delta_{j, j^{\prime}}\right)}{\left|j-j^{\prime}\right|}\right)^{\alpha} j j^{\prime} h_{0}^{-2} \max _{l}\left\|\left(F_{l}^{\prime}\right)^{-1}\right\|_{B_{1, \infty}^{\alpha}([k h,(k+1) h])}
$$

for the norm over $H_{n, l}^{2}$. Putting all estimates together gives

$$
\begin{aligned}
& \left\|\operatorname{COV}_{\mathcal{E}_{1}}\left(\mathrm{LMM}_{\text {or }}^{(n)}\right)-\operatorname{COV}_{\mathcal{E}_{2}}\left(\mathrm{LMM}_{\text {or }}^{(n)}\right)\right\| \\
& \lesssim h \sum_{j, j^{\prime}=1}^{\infty} h_{0}^{-2}\left(1+j^{\prime} / h_{0}\right)^{-4}\left(1+j / h_{0}\right)^{-2} h^{\alpha}\left(1+\left|j-j^{\prime}\right|\right)^{-\alpha}\left(1+j j^{\prime} h_{0}^{-2}\right) .
\end{aligned}
$$

By comparison with the double integral (in terms of $x \approx j / h_{0}, y \approx j^{\prime} / h_{0}$ )

$$
\int_{0}^{\infty} \int_{0}^{\infty}(1+y)^{-4}(1+x)^{-2}|x-y|^{-\alpha}(1+x y) d x d y \lesssim 1
$$

we conclude

$$
\left\|\operatorname{COV}_{\mathcal{E}_{1}}\left(\mathrm{LMM}_{\text {or }}^{(n)}\right)-\operatorname{COV}_{\mathcal{E}_{2}}\left(\mathrm{LMM}_{\text {or }}^{(n)}\right)\right\| \lesssim h n_{\text {min }}^{-\alpha / 2}
$$

Arguing exactly as in Section C.2 for the case of $\Sigma$ being a sum of a $B_{1, \infty^{-}}^{\alpha}$ function and an $L^{2}$-martingale, the difference of covariances is in general of order $\mathcal{O}\left(h n_{\text {min }}^{-\alpha / 2}\right)+\mathcal{O}_{P}\left(h^{2}\right)$.

C.4. Proof of Theorem 3.4. Let us denote the rate of convergence of $\hat{\Sigma}$ by $\delta_{n}=n_{\min }^{-\alpha /(4 \alpha+2)}$. For later use we note the order bounds

$$
\delta_{n}=\mathcal{O}\left(r^{1 / 2} h_{0}^{-1 / 2}\left(n_{\min } / n_{\max }\right)^{1 / 4}\right), \quad \delta_{n}=\mathcal{O}\left(h_{0}^{-1}\left(n_{\min } / n_{\max }\right)^{1 / 2}\right)
$$

First, we show that

$$
\left\|\mathrm{LMM}_{o r}^{(n)}-\mathrm{LMM}_{a d}^{(n)}\right\|=\mathcal{O}_{P}\left(n_{m a x}^{-1 / 4}\right)
$$


which by Slutsky's Lemma implies the CLT with normalisation matrix $\mathbf{I}_{\mathbf{n}}$. This in turn is already sufficient for obtaining the result of Corollary 3.3 for $\mathrm{LMM}_{a d}^{(n)}$. Let us start with proving that

$$
T_{n}^{m}:=\left\|\sum_{m=0}^{r^{-1}-1} h \sum_{k=m r / h}^{(m+1) r / h-1} \sum_{j=1}^{\infty}\left(W_{j}\left(\hat{\Sigma}^{m r}\right)-W_{j}\left(\Sigma^{m r}\right)\right) Z_{j k}\right\|=\mathcal{O}_{P}\left(n_{\max }^{-1 / 4}\right)
$$

where the random variables

$$
Z_{j k}=\operatorname{vec}\left(S_{j k} S_{j k}^{\top}-\pi^{2} j^{2} h^{-2} \operatorname{diag}\left(\left(H_{n, l}^{k h}\right)^{2}\right)_{1 \leq l \leq d}-\Sigma^{k h}\right)
$$

are independent, $\mathbb{E}_{\mathcal{E}_{2}}\left[Z_{j k}\right]=0, \mathbb{C O V}_{\mathcal{E}_{2}}\left(Z_{j k}\right)=I_{j k}^{-1} \mathcal{Z}$. We have

$$
T_{n}^{m} \leq \sum_{m=0}^{r^{-1}-1} h \sum_{j=1}^{\infty}\left\|W_{j}\left(\hat{\Sigma}^{m r}\right)-W_{j}\left(\Sigma^{m r}\right)\right\|\left\|\sum_{k=m r / h}^{(m+1) r / h-1} Z_{j k}\right\|
$$

since the weight matrices do not depend on $k$ on the same block of the coarse grid. Using Lemma C.2 and that $\|\hat{\Sigma}-\Sigma\|_{L^{1}}=\mathcal{O}_{P}\left(\delta_{n}\right)$, we obtain

$$
\begin{aligned}
\left\|W_{j}\left(\hat{\Sigma}^{m r}\right)-W_{j}\left(\Sigma^{m r}\right)\right\| & \leq \max _{k}\left\|\frac{d W_{j}\left(\Sigma^{k h}\right)}{d \Sigma^{k h}}\right\|\left\|\hat{\Sigma}^{m r}-\Sigma^{m r}\right\| \\
& =\mathcal{O}_{P}\left(\left(h_{0}^{-1} \wedge h_{0}^{3} j^{-4}\right) r^{-1}\|\hat{\Sigma}-\Sigma\|_{L^{1}([m r,(m+1) r])}\right) .
\end{aligned}
$$

For the second factor in (C.5) we employ $\left\|\mathbb{C O V}_{\mathcal{E}_{2}}\left(Z_{j k}\right)\right\|=2\left\|C_{j k}\right\|^{2}$ such that $\left\|Z_{j k}\right\|=\mathcal{O}_{P}\left(\left\|C_{j k}\right\|\right)$. Consequently, (C.3) implies for $T_{n}^{m}$ the bound

$$
\begin{aligned}
& \sum_{m=0}^{r^{-1}-1} h \sum_{j=1}^{\infty} \mathcal{O}_{P}\left(\left(h_{0}^{-1} \wedge h_{0}^{3} j^{-4}\right) r^{-1}\|\hat{\Sigma}-\Sigma\|_{L^{1}([m r,(m+1) r])}(r / h)^{1 / 2}\left(1 \vee j^{2} h_{0}^{-2}\right)\right) \\
& =\mathcal{O}_{P}\left(r^{-1 / 2} h^{1 / 2} \delta_{n}\right)=\mathcal{O}_{P}\left(n_{\max }^{-1 / 4}\right) .
\end{aligned}
$$

The asymptotics (C.4) follow if we can ensure that the coarse grid approximations of the weights induce a negligible error, i.e. if also

$$
\sum_{m=0}^{r^{-1}-1} \sum_{k=m r / h}^{(m+1) r / h-1} h^{2} \sum_{j=1}^{\infty}\left(W_{j}\left(\Sigma^{k h}\right)-W_{j}\left(\Sigma^{m r}\right)\right) Z_{j k}=\mathcal{O}_{P}\left(n_{\max }^{-1 / 4}\right)
$$

holds. The term is centred and its covariance matrix is bounded in norm by

$$
\sum_{m=0}^{r^{-1}-1} \sum_{k=m r / h}^{(m+1) r / h-1} h^{2} \sum_{j=1}^{\infty}\left\|W_{j}\left(\Sigma^{k h}\right)-W_{j}\left(\Sigma^{m r}\right)\right\|^{2}\left\|I_{j k}^{-1}\right\| .
$$


From Lemma C. $2,\left\|I_{j k}^{-1}\right\|=2\left\|C_{j k}\right\|^{2} \lesssim 1+j^{4} h_{0}^{-4}$ and $\Sigma \in B_{1, \infty}^{\alpha}([0,1])$ we derive the upper bound

$$
\mathcal{O}\left(\sum_{k=0}^{h^{-1}-1} h^{2} \sum_{j=1}^{\infty} r^{2} h_{0}^{-2}\left(1+j^{4} h_{0}^{-4}\right)^{-1}\right)=\mathcal{O}\left(n_{\min }^{-1 / 2} r^{2 \alpha}\right)=\mathcal{O}\left(n_{\max }^{-1 / 2}\right)
$$

by the choice of $r$ and $\alpha>1 / 2$.

Another application of Slutsky's Lemma yields the CLT with normalisation matrix $\hat{\mathbf{I}}_{n}$ provided $\mathbf{I}_{n}^{1 / 2} \hat{\mathbf{I}}_{n}^{-1 / 2} \rightarrow E_{d^{2}}$ in probability. The proof of Lemma C.2, more specifically the bound on the last term in (C.2), yields also

$$
\left\|\frac{d}{d \Sigma^{k h}} I_{j}\left(\Sigma^{k h}\right)\right\| \lesssim h_{0}^{-1}\left(1+j^{4} h_{0}^{-4}\right)^{-1} .
$$

This implies $\sum_{k, j}\left\|\hat{I}_{j k}-I_{j k}\right\|=\mathcal{O}_{P}\left(h^{-1} \delta_{n}\right)$. Using $\hat{A}^{-1}-A^{-1}=A^{-1}(\hat{A}-$ A) $\hat{A}^{-1}$ and $\left\|I_{k}^{-1}\right\| \lesssim h_{0}^{-1}$, we infer

$$
\left\|\hat{\mathbf{I}}_{n}^{-1}-\mathbf{I}_{n}^{-1}\right\| \leq \sum_{k=0}^{h^{-1}-1} h^{2}\left\|\left(\sum_{j=1}^{\infty} \hat{I}_{j k}\right)^{-1}-\left(\sum_{j=1}^{\infty} I_{j k}\right)^{-1}\right\|=\mathcal{O}_{P}\left(h \delta_{n} h_{0}^{-2}\right) .
$$

The smallest eigenvalue of $\mathbf{I}_{n}^{-1}$ equals $\left\|\mathbf{I}_{n}\right\|^{-1}$ which has order at least $n_{\text {max }}^{-1 / 2}$. The global Lipschitz constant $L_{n}$ of $f(x)=x^{1 / 2}$ for $x \geq\left\|\mathbf{I}_{n}\right\|^{-1}$ is therefore of order $n_{\text {max }}^{1 / 4}$. The perturbation result from [16] for functional calculus therefore implies

$$
\left\|\mathbf{I}_{n}^{1 / 2} \hat{\mathbf{I}}_{n}^{-1 / 2}-E_{d}\right\| \leq L_{n}\left\|\mathbf{I}_{n}^{1 / 2}\right\|\left\|\mathbf{I}_{n}^{-1}-\hat{\mathbf{I}}_{n}^{-1}\right\|=\mathcal{O}_{P}\left(n_{\max }^{1 / 2} h \delta_{n} h_{0}^{-2}\right) .
$$

The order is $\left(n_{\max } / n_{\min }\right)^{1 / 2} h_{0}^{-1} \delta_{n}$ and tends to zero by (C.3).

\section{APPENDIX D: PROOF OF THE LOWER BOUND}

D.1. Proof of Lemma 4.1. Since $\mathcal{M}_{\left(R^{\prime}\right)^{1 / 2}} T_{r}$ is an isometry on $L^{2}\left([0,1] ; \mathbb{R}^{d}\right)$, we obtain directly for the adjoint $T_{r}^{*}=T_{r}^{-1} \mathcal{M}_{\left(R^{\prime}\right)^{-1}}$. We observe in a formal differential notation:

$$
\begin{aligned}
T_{r}^{*} \mathcal{M}_{\left(R^{\prime}\right)^{1 / 2} O} d Y & =T_{r}^{-1} \mathcal{M}_{\left(R^{\prime}\right)^{-1 / 2} O}\left(X d t+\frac{1}{\sqrt{n}} d W\right) \\
& =-T_{r}^{-1} I^{*}\left(\mathcal{M}_{\left(\left(R^{\prime}\right)^{-1 / 2} O\right)^{\prime}} X d t+\mathcal{M}_{\left(R^{\prime}\right)^{-1 / 2} O} d X\right)+\frac{1}{\sqrt{n}} d \bar{W} \\
& =-I^{*} T_{r}^{*}\left(\mathcal{M}_{\left(\left(R^{\prime}\right)^{-1 / 2} O\right)^{\prime}} X d t+\mathcal{M}_{\left(R^{\prime}\right)^{-1 / 2} O} d X\right)+\frac{1}{\sqrt{n}} d \bar{W}
\end{aligned}
$$

Here, we use that $T_{r}^{*} \mathcal{M}_{\left(R^{\prime}\right)^{1 / 2} O}$ is an $L^{2}$-isometry and we introduce the independent Brownian motions $\bar{W}, \bar{B}$ via the differentials

$$
d \bar{W}=T_{r}^{*} \mathcal{M}_{\left(R^{\prime}\right)^{1 / 2} O} d W, \quad d \bar{B}=T_{r}^{*} \mathcal{M}_{\left(R^{\prime}\right)^{1 / 2} O} d B
$$


or alternatively $\left(\right.$ apply $\left.-I^{*}\right)$ via their coordinates $i=1, \ldots, d$ as

$$
\bar{W}_{i}(u)=\sum_{j=1}^{d} \int_{0}^{r_{i}^{-1}(u)} R_{i i}^{\prime}(s)^{1 / 2} O_{i j}(s) d W_{j}(s),
$$

and $\bar{B}_{i}(u)$ analogously.

The formal derivations are made rigorous by duality, that is testing stochastic differentials with deterministic $L^{2}$-functions. We infer from the coordinate-wise definition of $\bar{W}$ for $f \in L^{2}\left([0,1] ; \mathbb{R}^{d}\right)$ (e.g., check via indicator functions $f$ )

$$
\int_{0}^{1}\left\langle O(t)^{\top} R^{\prime}(t)^{1 / 2}\left(T_{r} f\right)(t), d W_{t}\right\rangle=\int_{0}^{1}\langle f(u), d \bar{W}(u)\rangle
$$

and equally for $\bar{B}$. Now consider for functions $g \in L^{2}\left([0,1] ; \mathbb{R}^{d}\right)$ the real observations

$$
\begin{aligned}
\int_{0}^{1}\left\langle O(t)^{\top} R^{\prime}(t)^{1 / 2}\left(T_{r} g\right)(t), d Y_{t}\right\rangle=\int_{0}^{1}\left\langle O(t)^{\top} R^{\prime}(t)^{-1 / 2}\left(T_{r} I g\right)^{\prime}(t), d Y_{t}\right\rangle \\
=\int_{0}^{1}\left\langle\left(O(t)^{\top} R^{\prime}(t)^{-1 / 2}\left(T_{r} I g\right)\right)^{\prime}-\left(O(t)^{\top} R^{\prime}(t)^{-1 / 2}\right)^{\prime}\left(T_{r} I g\right)(t), X_{t}\right\rangle d t \\
\quad+\frac{1}{\sqrt{n}} \int_{0}^{1}\left\langle O(t)^{\top} R^{\prime}(t)^{1 / 2}\left(T_{r} g\right)(t), d W_{t}\right\rangle \\
=\int_{0}^{1}\left\langle-\left(O(t)^{\top} R^{\prime}(t)^{-1 / 2}\right)^{\prime}\left(T_{r} I g\right)(t), X_{t}\right\rangle d t \\
\quad-\int_{0}^{1}\left\langle O(t)^{\top} R^{\prime}(t)^{-1 / 2}\left(T_{r} I g\right)(t), d X_{t}\right\rangle+\frac{1}{\sqrt{n}} \int_{0}^{1}\left\langle g(u), d \bar{W}_{u}\right\rangle \\
=\int_{0}^{1}\left\langle-\left(O(t)^{\top} R^{\prime}(t)^{-1 / 2}\right)^{\prime}\left(T_{r} I g\right)(t), X_{t}\right\rangle d t \\
\quad-\int_{0}^{1}\left\langle\Sigma(t)^{1 / 2} O(t)^{\top} R^{\prime}(t)^{-1 / 2}\left(T_{r} I g\right)(t), d B_{t}\right\rangle+\frac{1}{\sqrt{n}} \int_{0}^{1}\left\langle g(u), d \bar{W}_{u}\right\rangle .
\end{aligned}
$$

For $\varepsilon=0$ we use $\left(R^{\prime}\right)^{-1 / 2} \Lambda\left(R^{\prime}\right)^{-1 / 2}=\bar{\Lambda}$ and evaluate the first two terms of the last display as

$$
\int_{0}^{1}\left\langle-\left(O(t)^{\top} R^{\prime}(t)^{-1 / 2}\right)^{\prime}\left(T_{r} I g\right)(t), X_{t}\right\rangle d t-\int_{0}^{1}\left\langle\left(T_{r}^{-1} \bar{\Lambda}\left(T_{r} I g\right)\right)(u), d \bar{B}_{u}\right\rangle .
$$

As $\bar{\Lambda}$ is constant in time, the second term is equal to $-\int_{0}^{1}\langle I g, \bar{\Lambda} d \bar{B}\rangle$ and the formal derivations above are confirmed. 
D.2. Proof of Lemma 4.2. In a first step note that for general operators $A, B$ we have

$$
\begin{aligned}
\left\|A A^{*}-B B^{*}\right\|_{H S}^{2} & =\frac{1}{2}\left\|(2 A+B-A)(A-B)^{*}+(2 A+B-A)^{*}(A-B)\right\|_{H S} \\
& \leq 2\|A\|\|A-B\|_{H S}+\|A-B\|_{H S}^{2} .
\end{aligned}
$$

Hence, it suffices to show

$$
\left\|Q_{n, 0}^{-1 / 2} Q_{n, 1}^{1 / 2}\right\| \lesssim 1 \text { and }\left\|Q_{n, 0}^{-1 / 2} Q_{n, 1}^{1 / 2}-C_{n, 0}^{-1 / 2} C_{n, 1}^{1 / 2}\right\|_{H S} \lesssim 1
$$

A further reduction is achieved by splitting terms to obtain

$$
\begin{aligned}
\left\|Q_{n, 0}^{-1 / 2} Q_{n, 1}^{1 / 2}-C_{n, 0}^{-1 / 2} C_{n, 1}^{1 / 2}\right\|_{H S} \leq\left\|\operatorname{Id}-C_{n, 0}^{-1 / 2} Q_{n, 0}^{1 / 2}\right\|_{H S}\left\|Q_{n, 0}^{-1 / 2} Q_{n, 1}^{1 / 2}\right\| \\
+\left\|C_{n, 0}^{-1 / 2} Q_{n, 0}^{1 / 2}\right\|\left\|Q_{n, 0}^{-1 / 2} Q_{n, 1}^{1 / 2}\right\|\left\|\operatorname{Id}-Q_{n, 1}^{-1 / 2} C_{n, 1}^{1 / 2}\right\|_{H S} .
\end{aligned}
$$

Owing to $\left\|C_{n, 0}^{-1 / 2} Q_{n, 0}^{1 / 2}\right\| \leq 1+\left\|\mathrm{Id}-C_{n, 0}^{-1 / 2} Q_{n, 0}^{1 / 2}\right\|_{H S}$ it remains to show $\left\|Q_{n, 0}^{-1 / 2} Q_{n, 1}^{1 / 2}\right\| \lesssim 1, \quad\left\|\operatorname{Id}-C_{n, 0}^{-1 / 2} Q_{n, 0}^{1 / 2}\right\|_{H S} \lesssim 1$ and $\left\|\operatorname{Id}-Q_{n, 0}^{-1 / 2} C_{n, 0}^{1 / 2}\right\|_{H S} \lesssim 1$.

Finally, we can use $Q_{n, 1}-Q_{n, 0}=Q_{\infty, 1}-Q_{\infty, 0}, Q_{n, 1} \geq Q_{\infty, 1}$ in operator order (and similarly for $C_{n, \varepsilon}$ ) as well as $|a-1| \leq\left|a^{2}-1\right|$ for $a \geq 0$ implying $\|A-\mathrm{Id}\|_{H S} \leq\left\|A A^{*}-\mathrm{Id}\right\|_{H S}$ for positive operators $A$. We are thus left with proving that the following three quantities are uniformly bounded

$\left\|Q_{n, 0}^{-1 / 2} Q_{n, 1}^{1 / 2}\right\|,\left\|C_{\infty, 0}^{-1 / 2}\left(Q_{\infty, 0}-C_{\infty, 0}\right) C_{\infty, 0}^{-1 / 2}\right\|_{H S},\left\|Q_{\infty, 1}^{-1 / 2}\left(C_{\infty, 1}^{1 / 2}-Q_{\infty, 1}^{1 / 2}\right) Q_{\infty, 1}^{-1 / 2}\right\|_{H S}$.

By the Feldman-Hajek Theorem for Gaussian measures, see e.g. [8], the latter two quantities are finite iff the Gaussian laws $\mathbf{N}\left(0, C_{\infty, \varepsilon}\right)$ and $\mathbf{N}\left(0, Q_{\infty, \varepsilon}\right)$, are equivalent for $\varepsilon \in\{0,1\}$. Using again differential notation, these are the laws of

$$
Z^{C}:=T_{r}^{*} \mathcal{M}_{\left(R^{\prime}\right)^{1 / 2} O} X, \quad Z^{Q}:=-I^{*} T_{r}^{*} \mathcal{M}_{\left(R^{\prime}\right)^{-1 / 2} O} d X
$$

where $d X=\Sigma^{1 / 2} d B$ for the $\varepsilon$ at hand. Both processes are images in $C\left([0,1], \mathbb{R}^{d}\right)$ under the linear (and thus measurable) map $T_{r}^{-1}=T_{r}^{*} \mathcal{M}_{R^{\prime}}$ of the respective processes

$$
\tilde{Z}^{C}:=\mathcal{M}_{\left(R^{\prime}\right)^{-1 / 2} O} X, \quad \tilde{Z}^{Q}:=-I^{*} \mathcal{M}_{\left(R^{\prime}\right)^{-1 / 2} O} d X .
$$

By the product rule we see

$$
\begin{aligned}
\tilde{Z}^{C}(t) & =-I^{*}\left\{\mathcal{M}_{\left(R^{\prime}\right)^{-1 / 2} O} d X+\mathcal{M}_{\left(\left(R^{\prime}\right)^{-1 / 2} O\right)^{\prime}} X\right\}(t) \\
& =\tilde{Z}^{Q}(t)+\int_{0}^{t}\left(\left(R^{\prime}\right)^{-1 / 2} O\right)^{\prime}(s) X(s) d s .
\end{aligned}
$$


Hence, $\tilde{Z}^{C}$ equals the Brownian martingale $\tilde{Z}^{Q}$ plus an adapted linear drift in $X$. By Girsanov's theorem, noting that all deterministic quantities are continuous and bounded away from zero, the laws of $\tilde{Z}^{C}$ and $\tilde{Z}^{Q}$ are equivalent, e.g. use Thm. 3.5.1. together with Cor. 3.5.16 in [15]. Hence, so are the laws of their images $Z^{C}$ and $Z^{Q}$, as required.

Let us finally consider $Q_{n, 0}^{-1 / 2} Q_{n, 1}^{1 / 2}$. Its squared norm equals

$$
\begin{aligned}
\sup _{f \in L^{2}} \frac{\left\langle Q_{n, 1} f, f\right\rangle}{\left\langle Q_{n, 0} f, f\right\rangle} & =\sup _{f \in L^{2}} \frac{\left\langle\mathcal{M}_{\left.\left(R^{\prime}\right)^{-1 / 2} O \Sigma_{1} O^{\top}\left(R^{\prime}\right)^{-1 / 2} T_{r} I f, T_{r} I f\right\rangle+\frac{1}{n}\|f\|^{2}}\right.}{\|I f\|^{2}+\frac{1}{n}\|f\|^{2}} \\
& \leq\left(1+\|M\|_{\infty}\right) \max _{i=1, \ldots, d}\left\|\left(r_{i}^{-1}\right)^{\prime}\right\|_{\infty} .
\end{aligned}
$$

This uniform bound is finite under our regularity assumptions.

D.3. Proof of Theorem 4.3. Without loss of generality we may assume that $A(t)$ is symmetric for all $t$ because $\Sigma(t)$ is symmetric. Owing to $\mathcal{Z} \operatorname{vec}(A)=2 \operatorname{vec}(A)$ and $\left(\Sigma_{0} \otimes \Sigma_{0}^{1 / 2}\right) \operatorname{vec}(A)=\operatorname{vec}\left(\Sigma_{0}^{1 / 2} A \Sigma_{0}\right)$, we thus have to show in terms of the Hilbert-Schmidt scalar product

$$
\left.\operatorname{Var}_{\varepsilon=0}\left(\hat{\vartheta}_{n}\right) \geq \frac{(8+\mathcal{O}(1))}{\sqrt{n}} \int_{0}^{1}\left\langle\left(\Sigma_{0} \otimes \Sigma_{0}^{1 / 2}+\Sigma_{0}^{1 / 2} \otimes \Sigma_{0}\right) A\right)(t), A(t)\right\rangle_{H S} d t .
$$

Since $C_{B M}$ is a positive operator on $L^{2}([0,1] ; \mathbb{R})$, we can define the bounded self-adjoint operator

$$
\Delta_{n}^{\sigma}=I\left(\sigma^{2} C_{B M}+\frac{1}{n} \mathrm{Id}\right)^{-1} I^{*}=\left(\sigma^{2} \mathrm{Id}+\frac{1}{n} C_{B M}^{-1}\right)^{-1} .
$$

$C_{B M}$ is Hilbert-Schmidt and so is $\Delta_{n}^{\sigma}$. We identify its kernel $\delta_{n}^{\sigma}:[0,1]^{2} \rightarrow \mathbb{R}$ (or Green function) as

$$
\delta_{n}^{\sigma}(t, s)=\frac{\sqrt{n}}{2 \sigma \cosh (\sigma \sqrt{n})}(\sinh (\sigma \sqrt{n}(1-|s-t|))+\sinh (\sigma \sqrt{n}(t+s-1))) .
$$

This can be formally derived from the properties $C_{B M}^{-1}=-D^{2}$ on its domain, $\delta_{n}^{\sigma}$ in the domain (i.e. $\delta_{n}^{\sigma}(0, s)=0,\left(\delta_{n}^{\sigma}\right)^{\prime}(1, s)=0$ ) and $\delta_{n}^{\sigma}(t, s)=\Delta_{n}^{\sigma} \delta_{s}(t)$. Alternatively use the eigenvalue-eigenfunction decomposition of $C_{B M}$ and apply functional calculus. The main observation is that $\delta_{n}^{\sigma}$ has all the properties of a smoothing kernel, which for $n \rightarrow \infty$ concentrates on the diagonal $\{t=s\}$, where it approximates the uniform law. This is best seen by the approximation for large $n$

$\delta_{n}^{\sigma}(t, s) \asymp \frac{\sqrt{n}}{2 \sigma}(\exp (-\sigma \sqrt{n}|s-t|)+\operatorname{sgn}(t+s-1) \exp (\sigma \sqrt{n}(|t+s-1|-1)))$, 
observing $|t+s-1|-1 \leq|s-t|+|2 t-1|-1$ such that the second exponential asymptotically only contributes at the corners $(0,0)$ and $(1,1)$ of the diagonal.

We shall see, however, that for the Hilbert-Schmidt norm evaluation we face $\left(\delta_{n}^{\sigma}\right)^{2}$ as the operator kernel, which also behaves like a smoothing kernel on the diagonal, but needs to be rescaled by $\left\|\delta_{n}^{\sigma}\right\|_{L^{2}}^{2}=(1+\mathcal{O}(1)) \sqrt{n} /\left(4 \sigma^{3}\right)$.

Consequently, in terms of $\Delta_{n}=\operatorname{diag}\left(\Delta_{n}^{\bar{\Lambda}_{i i}}\right)_{i=1, \ldots, d}$ and its kernel $\delta_{n}(t, s)=$ $\operatorname{diag}\left(\delta_{n}^{\bar{\Lambda}_{i i}}(t, s)\right)_{i=1, \ldots, d}$, the Fisher information evaluates as

$$
\begin{aligned}
I_{n}^{Q} & =\frac{1}{2}\left\|Q_{n, 0}^{-1 / 2} \dot{Q}_{0} Q_{n, 0}^{-1 / 2}\right\|_{H S}^{2} \\
& =\frac{1}{2} \operatorname{trace}\left(T_{r} I Q_{n, 0}^{-1} I^{*} T_{r}^{*} \mathcal{M}_{M} T_{r} I Q_{n, 0}^{-1} I^{*} T_{r}^{*} \mathcal{M}_{M}\right) \\
& =\frac{1}{2} \operatorname{trace}\left(\left(T_{r} \Delta_{n} T_{r}^{*}\right) \mathcal{M}_{M}\left(T_{r} \Delta_{n} T_{r}^{*}\right) \mathcal{M}_{M}\right) \\
& =\frac{1}{2} \int_{0}^{1} \int_{0}^{1} \operatorname{trace}_{\mathbb{R}^{d \times d}}\left(\delta_{n}(r(t), r(s)) M(s) \delta_{n}(r(s), r(t)) M(t)\right) d t d s .
\end{aligned}
$$

We now use $\int_{0}^{1} M(s) a_{n} e^{-|t-s| a_{n}} d s=2 M(t)(1+\mathcal{O}(1))$ uniformly over $t \in$ $\left[b_{n}, 1-b_{n}\right]$ whenever $a_{n} \rightarrow \infty, a_{n} b_{n} \rightarrow \infty$ and $M(t)$ is continuously differentiable. Together with the asymptotic behaviour of $\delta_{n}^{\sigma}$ we obtain

$$
\begin{aligned}
& \int_{0}^{1} \delta_{n}^{\bar{\Lambda}_{i i}}\left(r_{i}(t), r_{i}(s)\right) M_{i j}(s) \delta_{n}^{\bar{\Lambda}_{j j}}\left(r_{j}(s), r_{j}(t)\right) d s \\
& =(1+\mathcal{O}(1)) \frac{n}{4 \bar{\Lambda}_{i i} \bar{\Lambda}_{j j}} \int_{0}^{1} \exp \left(-\sqrt{n}\left(\bar{\Lambda}_{i i} r_{i}^{\prime}(t)+\bar{\Lambda}_{j j} r_{j}^{\prime}(t)\right)|t-s|\right) M_{i j}(s) d s \\
& =(1+\mathcal{O}(1)) \frac{\sqrt{n}}{2 \bar{\Lambda}_{i i} \bar{\Lambda}_{j j}\left(\bar{\Lambda}_{i i} r_{i}^{\prime}(t)+\bar{\Lambda}_{j j} r_{j}^{\prime}(t)\right)} M_{i j}(t) \\
& =\frac{\sqrt{n} M_{i j}(t)(1+\mathcal{O}(1))}{2 \bar{\Lambda}_{i i} \bar{\Lambda}_{j j}\left(\lambda_{i}(t)+\lambda_{j}(t)\right)}
\end{aligned}
$$

with $\mathcal{O}(1)$ uniformly in $n$ and $t \in\left[n^{-p}, 1-n^{-p}\right]$ for any $p \in(0,1 / 2)$ to infer

$$
\begin{aligned}
I_{n}^{Q} & =\frac{\sqrt{n}}{4} \sum_{i, j=1}^{d} \bar{\Lambda}_{i i}^{-1} \bar{\Lambda}_{j j}^{-1} \int_{0}^{1}\left(\lambda_{i}(t)+\lambda_{j}(t)\right)^{-1} M_{i j}(t)^{2}(1+\mathcal{O}(1)) d t \\
& =\frac{\sqrt{n}(1+\mathcal{O}(1))}{4} \int_{0}^{1} \sum_{i, j=1}^{d} \frac{\left(O \mathbb{H} O^{\top}\right)_{i j}^{2}}{\lambda_{i}\left(\lambda_{i}+\lambda_{j}\right) \lambda_{j}}(t) d t .
\end{aligned}
$$

Asymptotically for $n \rightarrow \infty$ neglecting terms of smaller order, this bound is obtained by the worst parametric perturbation $\mathbb{H}^{*}(t)=\Sigma_{0} A \Sigma_{0}^{1 / 2}+$ 
$\Sigma_{0}^{1 / 2} A \Sigma_{0}$, which we evaluate using duality with respect to the scalar product $\int_{0}^{1} \sum_{i, j} A_{i j}(t) B_{i j}(t) d t$ as

$$
\begin{aligned}
& \sup _{\mathbb{H}: \mathbb{H}(t)=\mathbb{H}(t)^{\top}} \frac{\left(\int_{0}^{1} \sum_{i, j=1}^{d} A_{i j}(t) \mathbb{H}_{i j}(t) d t\right)^{2}}{\frac{\sqrt{n}}{4} \int_{0}^{1} \sum_{k, l=1}^{d} \frac{\left(O H O^{\top}\right)_{k l}^{2}}{\lambda_{k}\left(\lambda_{k}+\lambda_{l}\right) \lambda_{l}}(t) d t}=\frac{\left(\int_{0}^{1} \sum_{i, j=1}^{d} A_{i j}(t) \mathbb{H}_{i j}^{*}(t) d t\right)^{2}}{\frac{\sqrt{n}}{4} \int_{0}^{1} \sum_{k, l=1}^{d} \frac{\left(O H^{*} O^{\top}\right)_{k l}^{2}}{\lambda_{k}\left(\lambda_{k}+\lambda_{l}\right) \lambda_{l}}(t) d t} \\
& =\frac{4}{\sqrt{n}} \int_{0}^{1}\left\langle A(t),\left(\Sigma A \Sigma^{1 / 2}+\Sigma^{1 / 2} A \Sigma\right)(t)\right\rangle_{H S} d t .
\end{aligned}
$$

Finally, remark that the Cramér-Rao inequality, e.g. [18, Thm. 2.5.10], is applicable since $\left(\mathbf{N}\left(0, Q_{n, \varepsilon}\right)\right)_{\varepsilon}$ forms an exponential family in $\left(Q_{n, \varepsilon}^{-1}\right)_{\varepsilon}$, which is differentiable at $\varepsilon=0$, and thus the models $\left(\mathbf{N}\left(0, Q_{n, \varepsilon}\right)\right)_{\varepsilon}$ as well as $\left(\mathbf{N}\left(0, C_{n, \varepsilon}\right)\right)_{\varepsilon}$ are regular.

\section{REFERENCES}

[1] Aїт-Sahalia, Y. and Fan, J. and XiU, D. (2010). High frequency covariance estimates with noisy and asynchronous financial data. J. Amer. Statist. Assoc. 105 1504-1517. MR2796567

[2] Andersen, T. and Bollerslev, T. (1997). Intraday perdiodicity and volatility persistence in financial markets. J. Empir. Financ. 4 115-158.

[3] Barndorff-Nielsen, O. E. and Hansen, P. R. and Lunde, A. and Shephard, N. (2011). Multivariate realised kernels: consistent positive semi-definite estimators of the covariation of equity prices with noise and non-synchronous trading. J. Econometrics 162(2) 149-169.

[4] Bibinger, M. and Reiss, M. (2013). Spectral estimation of covolatility from noisy observations using local weights. Scand. J. Stat., to appear.

[5] Christensen, K. and Podolskij, M. and Vetter, M. (2012). On covariation estimation for multivariate continuous Itô semimartingales with noise in non-synchronous observation schemes. Preprint.

[6] Ciesielski, Z., Kerkyacharian, G. and Roynette, B. (1993). Quelques espaces fonctionnels associés à des processus gaussiens, Studia Math. 107(2), 171-203. MR1244574

[7] Cohen, A. (2003). Numerical Analysis of Wavelet Methods, Studies in Math. Appl. 32, Elsevier. MR1990555

[8] Da Prato, G. And Zabczyk, J. (2008). Stochastic Equations in Infinite Dimensions, corr. ed., Cambridge University Press. MR1207136

[9] FACKLeR, P. L. (2005). Notes on matrix calculus, Lecture Notes, North Carolina State University. http://www4.ncsu. edu/ pfackler/MatCalc.pdf

[10] Gloter, A. and Jacod, J. (2001). Diffusions with measurement errors 1 and 2. ESAIM, Probab. Stat. 5 225-242. MR1875672 \& 1875673

[11] Hansen, L. P. (1982). Large sample properties of generalized methods of moment estimation. Econometrica 50(4) 1029-1054.

[12] Hayashi, T. and Yoshida, N. (2011). Nonsynchronous covariation process and limit theorems. Stochastic Processes Appl. 121(10) 2416-2454. MR2822782

[13] Isserlis, L. (1918). On a formula for the product-moment coefficient of any order of a normal frequency distribution in any number of variables. Biometrika 12 134-139. 
[14] Kallenberg, O. (2002), Foundations of Modern Probability, 2nd ed., Springer. MR1876169

[15] Karatzas, I. And Shreve, S. E. (1991). Brownian Motion and Stochastic Calculus, 2nd ed., Springer Graduate Texts 113. MR1121940

[16] Kittaneh, F. (1985). On Lipschitz functions of normal operators. Proc. Amer. Math. Soc. 94, 416-418. MR0787884

[17] Le Cam, L. And Yang, L. G. (2000). Asymptotics in Statistics. Some basic concepts, 2nd ed., Springer. MR1784901

[18] Lehmann, E. L. And Casella, G. (1998). Theory of Point Estimation, 2nd ed., Springer. MR1639875

[19] ReIss, M. (2011). Asymptotic equivalence for inference on the volatility from noisy observations. Ann. Stat. 2 772-802. MR2816338

[20] Zhang, L. and Mykland, P. A. and Aït-Sahalia, Y. (2005). A Tale of Two Time Scales: Determining Integrated Volatility With Noisy High-Frequency Data. J. Amer. Statist. Assoc. 100 1394-1411. MR2236450

INSTITUT FÜR MATHEMATIK

Humboldt-UNiversität ZU BERLIN

UNTER DEN LINDEN 6

10099 Berlin, Germany

E-MAIL: bibinger@math.hu-berlin.de

School of Business and ECONOMics

Humboldt-Universität ZU BerLiN

SPANDAUER Str. 1

10178 BerLin, Germany

E-MAIL: malecpet@hu-berlin.de
SChOOL OF Business and ECONOMics

Humboldt-Universität ZU BerLin

SPANDAUER Str. 1

10178 Berlin, Germany

E-MAIL: nikolaus.hautsch@wiwi.hu-berlin.de INSTITUT FÜR MATHEMATIK Humboldt-Universität ZU BerLiN UNTER DEN LINDEN 6 10099 Berlin, Germany

E-MAIL: mreiss@math.hu-berlin.de 


\section{SFB 649 Discussion Paper Series 2013}

For a complete list of Discussion Papers published by the SFB 649, please visit http://sfb649. wiwi.hu-berlin.de.

001 "Functional Data Analysis of Generalized Quantile Regressions" by Mengmeng Guo, Lhan Zhou, Jianhua Z. Huang and Wolfgang Karl Härdle, January 2013.

002 "Statistical properties and stability of ratings in a subset of US firms" by Alexander B. Matthies, J anuary 2013.

003 "Empirical Research on Corporate Credit-Ratings: A Literature Review" by Alexander B. Matthies, January 2013.

004 "Preference for Randomization: Empirical and Experimental Evidence" by Nadja Dwenger, Dorothea Kübler and Georg Weizsäcker, January 2013.

005 "Pricing Rainfall Derivatives at the CME" by Brenda López Cabrera, Martin Odening and Matthias Ritter, January 2013.

006 "Inference for Multi-Dimensional High-Frequency Data: Equivalence of Methods, Central Limit Theorems, and an Application to Conditional Independence Testing" by Markus Bibinger and Per A. Mykland, January 2013.

007 "Crossing Network versus Dealer Market: Unique Equilibrium in the Allocation of Order Flow" by Jutta Dönges, Frank Heinemann and Tijmen R. Daniëls, J anuary 2013.

008 "Forecasting systemic impact in financial networks" by Nikolaus Hautsch, Julia Schaumburg and Melanie Schienle, January 2013.

009 "'I'll do it by myself as I knew it all along': On the failure of hindsightbiased principals to delegate optimally" by David Danz, Frank Hüber, Dorothea Kübler, Lydia Mechtenberg and Julia Schmid, January 2013.

010 "Composite Quantile Regression for the Single-Index Model" by Yan Fan, Wolfgang Karl Härdle, Weining Wang and Lixing Zhu, February 2013.

011 "The Real Consequences of Financial Stress" by Stefan Mittnik and Willi Semmler, February 2013.

012 "Are There Bubbles in the Sterling-dollar Exchange Rate? New Evidence from Sequential ADF Tests" by Timo Bettendorf and Wenjuan Chen, February 2013.

013 "A Transfer Mechanism for a Monetary Union" by Philipp Engler and Simon Voigts, March 2013.

014 "Do High-Frequency Data Improve High-Dimensional Portfolio Allocations?" by Nikolaus Hautsch, Lada M. Kyj and Peter Malec, March 2013.

015 "Cyclical Variation in Labor Hours and Productivity Using the ATUS" by Michael C. Burda, Daniel S. Hamermesh and Jay Stewart, March 2013.

016 "Quantitative forward guidance and the predictability of monetary policy - A wavelet based jump detection approach -" by Lars Winkelmann, April 2013.

017 "Estimating the Quadratic Covariation Matrix from Noisy Observations: Local Method of Moments and Efficiency" by Markus Bibinger, Nikolaus Hautsch, Peter Malec and Markus Reiss, April 2013.

\section{SFB 649, Spandauer Straße 1, D-10178 Berlin} http:/ / sfb649.wiwi.hu-berlin.de 